\title{
A Doppler Radar Emulator with an Application to the Detectability of Tornadic Signatures
}

\author{
Ryan M. May and Michael I. BiggerstafF \\ School of Meteorology, University of Oklahoma, Norman, Oklahoma \\ Ming Xue \\ School of Meteorology, and Center for Analysis and Prediction of Storms, University of Oklahoma, Norman, Oklahoma
}

(Manuscript received 14 June 2006, in final form 3 April 2007)

\begin{abstract}
A Doppler radar emulator was developed to simulate the expected mean returns from scanning radar, including pulse-to-pulse variability associated with changes in viewing angle and atmospheric structure. Based on the user's configuration, the emulator samples the numerical simulation output to produce simulated returned power, equivalent radar reflectivity, Doppler velocity, and Doppler spectrum width. The emulator is used to evaluate the impact of azimuthal over- and undersampling, gate spacing, velocity and range aliasing, antenna beamwidth and sidelobes, nonstandard (anomalous) pulse propagation, and wavelength-dependent Rayleigh attenuation on features of interest.

As an example, the emulator is used to evaluate the detection of the circulation associated with a tornado simulated within a supercell thunderstorm by the Advanced Regional Prediction System (ARPS). Several metrics for tornado intensity are examined, including peak Doppler velocity and axisymmetric vorticity, to determine the degradation of the tornadic signature as a function of range and azimuthal sampling intervals. For the case of a $2^{\circ}$ half-power beamwidth radar, like those deployed in the first integrated project of the Center for Collaborative Adaptive Sensing of the Atmosphere (CASA), the detection of the cyclonic shear associated with this simulated tornado will be difficult beyond the $10-\mathrm{km}$ range, if standard metrics such as azimuthal gate-to-gate shear from a single radar are used for detection.
\end{abstract}

\section{Introduction}

The design of a weather radar system and its scanning strategy involves trade-offs based upon features to be observed and the cost of building and deploying the radar system. Design trade-offs are often difficult to quantify in terms of their impacts on detecting and tracking features of interest. Moreover, the development of optimal scanning and the refinement of radarbased algorithms require large datasets to test the full range of environmental conditions and radar operating parameters to yield robust results. Recent advances in numerical modeling have made it possible to simulate convective storms at very fine scales over a broad range of environmental conditions (e.g., Wicker and Wil-

Corresponding author address: Ryan May, National Weather Center, 120 David L. Boren Blvd., Suite 5900, Norman, OK 73072-7307.

E-mail: rmay@ou.edu helmson 1995; Lewellen et al. 1997). Coupling a software radar emulator with high-resolution numerical simulations, one can generate large sets of simulated radar data that span a wide range of radar operating characteristics. These simulated datasets can be used to quantify the impact of radar design and operational mode on the diagnosis of storm features by automated algorithms.

Many approaches have been taken previously in simulating radar data, varying in sophistication from simple time series simulation (Zrnic 1975) to reflectivity calculation (Chandrasekar and Bringi 1987; Krajewski et al. 1993) to full simulation of radar returns from each pulse (Capsoni and D'Amico 1998; Capsoni et al. 2001). Zrnic (1975) generated simulated time series radar data and Doppler spectra using an assumed Gaussian distribution of velocities within the resolution volume. Chandrasekar and Bringi (1987) looked at the variation of simulated reflectivity values as a function of raindrop size distribution parameters. Similarly, Kra-

DOI: $10.1175 / 2007 J T E C H A 882.1$ 
jewski et al. (1993) calculated values of reflectivity factor and differential reflectivity using rainfall rates from a numerical model, with an assumed drop size distribution. Neither of these studies was concerned with Doppler velocity or the impacts of scanning strategies. Wood and Brown (1997) evaluated the effects of Weather Surveillance Radar-1988 Doppler (WSR-88D; Crum and Alberty 1993) scanning strategies on the sampling of mesocyclones and tornadoes. The effects of the scanning strategy were accounted for by using an effective beamwidth for the radar, which was used to scan an analytic vortex with a uniform reflectivity field. Capsoni and D'Amico (1998) simulated the pulse-topulse time series of radar data by combining the simulated returns from individual hydrometeors within a radar volume. This work was extended to generate polarimetric signatures by Capsoni et al. (2001). Because of the computational requirements of this approach, the radar data were generated for only a single range gate only, and thus many aspects of the scanning radar were not simulated.

This work describes a radar emulator designed to simulate the expected average returns from a scanning Doppler radar. Starting with output from a highresolution numerical simulation, the emulator generates fields of power, equivalent reflectivity factor, Doppler velocity, and Doppler spectrum width based on the radar configuration and scanning strategy used. Here we show that the emulator is capable of simulating several radar data characteristics, including range resolution, azimuthal over- and undersampling, nonstandard (anomalous) propagation, Rayleigh attenuation, antenna sidelobes, velocity aliasing, and range aliasing.

As an example of its use for research, the emulator is applied to output from a numerical simulation of an F3 intensity (Fujita 1971) supercell tornado simulated by the Advanced Regional Prediction System (ARPS; Xue et al. 2000, 2001) to evaluate the ability of $2^{\circ}$ beamwidth radars to directly detect the circulation associated with the tornado. This application is motivated by the first integrated project of the Center for Collaborative Adaptive Sensing of the Atmosphere (CASA; Brotzge et al. 2005), which recently deployed four such radars in the Oklahoma test bed.

\section{Radar emulator design}

\section{a. Emulator configuration and input}

The behavior of the radar emulator is controlled by specifying radar characteristics and scanning strategy (Table 1). Note that the antenna beamwidth, gain, and wavelength are treated independently to allow for vari-
TABLE 1. Emulator control parameters.

\begin{tabular}{ll}
\hline \multicolumn{1}{c}{ Radar parameters } & \multicolumn{1}{c}{$\begin{array}{c}\text { Scanning strategy } \\
\text { parameters }\end{array}$} \\
\hline Location & PRT \\
Antenna beamwidth & Pulse length \\
Antenna gain (including sidelobes) & Antenna rotation rate \\
Wavelength & No. of pulses per radial \\
Transmit power & Radar gate spacing \\
Range to first gate & Scan fixed angle \\
Minimum detectable signal & Scan start and end angles \\
\hline
\end{tabular}

ous types of antennas. The minimum detectable signal is used as a threshold to compensate for the lack of incorporation of noise (subgrid-scale turbulence and hardware electronic signals) on the quality of the emulated radar measurements. Hence, regions where signal-to-noise ratios would be expected to be low are deleted. The pulse repetition time and pulse length are given independently, but, in reality, they are usually constrained by the duty cycle of the transmitter. The antenna pointing angles can be specified for either full or sector plan position indicator (PPI) scans or rangeheight indicator (RHI) scans. The emulator allows for oversampling in both azimuth (or elevation for RHI scans) and range.

The input data to the radar emulator are threedimensional gridded fields that describe the state of the atmosphere. Wind components and mixing ratios of relevant precipitation-sized hydrometeors are required fields. Other water species can be included and used for additional scattering or attenuation. Water vapor, along with temperature and pressure, are needed for calculating the atmospheric index of refraction, which is used for anomalous propagation. Temperature is also used in determining the backscatter cross section of hydrometeors.

\section{b. Scattering}

For computational efficiency, backscattering and extinction cross sections per unit volume of air are precalculated at each model grid point. Moreover, only Rayleigh scattering by liquid hydrometeors is currently included. According to Battan (1973) and Doviak and Zrnic (1993), the Rayleigh approximation implies the following relationships for the backscattering cross section $\sigma_{b}$ and the extinction cross section $\sigma_{e}$ of a sphere of liquid water with diameter $D$ :

$$
\begin{aligned}
\sigma_{b} & \approx \frac{\pi^{5}}{\lambda^{4}}\left|K_{w}\right|^{2} D^{6}, \\
\sigma_{e} & \approx \frac{\pi^{2} D^{3}}{\lambda} \operatorname{Im}\left(-K_{w}\right)+\frac{2}{3} \sigma_{b},
\end{aligned}
$$




$$
K_{w}=\frac{m^{2}-1}{m^{2}+2},
$$

where $\lambda$ is radar wavelength, and $m$ is the complex index of refraction for liquid water. The emulator currently assumes a monodisperse distribution of cloud droplets and a Marshall and Palmer (1948) distribution of raindrops, which is consistent with the microphysics scheme used in many numerical models, including the ARPS. The use of a more general gamma distribution (Ulbrich 1983) would be possible. These assumed distributions permit the calculation of the total backscattering and extinction cross sections per unit volume from the cloud water $\rho_{c}$, and rainwater $\rho_{r}$ concentrations (in $\mathrm{kg} \mathrm{m}^{-3}$ ) at each grid point:

$$
\begin{aligned}
& \frac{\sigma_{b}}{V}=\frac{\pi^{5}}{\lambda^{4}}\left|K_{w}\right|^{2}\left[6 ! N_{0}\left(\frac{\rho_{r}}{\pi \rho_{l} N_{0}}\right)^{(7 / 4)}+\frac{48}{\pi \rho_{l}} R_{m}^{3} \rho_{c}\right], \\
& \frac{\sigma_{e}}{V}=\frac{6 \pi}{\lambda \rho_{l}} \operatorname{Im}\left(-K_{w}\right)\left(\rho_{r}-\rho_{c}\right),
\end{aligned}
$$

where $\rho_{l}$ is the density of liquid water; $R_{m}$ is the median radius for the cloud droplets; and $N_{0}$ is the MarshallPalmer distribution intercept parameter; $R_{m}$ and $N_{0}$ are assumed to have values of $50 \mu \mathrm{m}$ and 8000 drops $\mathrm{m}^{-3}$ $\mathrm{mm}^{-1}$, respectively. The Debye formula, shown by Saxton $(1946,278-325)$ to be applicable for the microwave region, is used to calculate the complex index of refraction explicitly. The Debye formula is

$$
m^{2}=\frac{\varepsilon_{1}-\varepsilon_{2}}{1+i\left(\frac{\lambda_{0}}{\lambda}\right)}+\varepsilon_{2},
$$

where $\varepsilon_{1}$ is the static dielectric constant, $\varepsilon_{2}$ is the optical dielectric constant, $\lambda_{0}$ is the transition wavelength, and $i$ equals $\sqrt{-1}$. Values of $\lambda_{0}, \varepsilon_{1}$, and $\varepsilon_{2}$ as a function of temperature were taken from Kerr (1951) and are based on Ryde and Ryde (1945). This formulation of $\mathrm{m}^{2}$ allows the emulator to capture the temperature and wavelength dependencies of $K_{w}$.

It is important to note that the Rayleigh approximation has been assumed for both scattering and attenuation. The range where the Rayleigh approximation is accurate for attenuation is much more limited than that for backscatter (Battan 1973). Therefore, at wavelengths shorter than approximately $10 \mathrm{~cm}$, the attenuation simulated here will grossly underestimate the actual attenuation. This limitation will be addressed in future work by using scattering parameters calculated using Mie theory for spherical scatterers (Mie 1908) and/or the T-matrix method for nonspherical scatterers (Waterman 1965).

\section{c. Sampling of input fields}

To sample the virtual model atmosphere, the emulator calculates radar variables along the path of individual pulses at the interval specified by the pulse repetition time (PRT). This allows the input model fields, as well as the state of the radar (such as antenna pointing angle), to change for individual pulses. While the emulator is currently configured for a mechanically scanning antenna, pulse-by-pulse calculation can be used to emulate measurements for phased-array radar as well. The pulse generated within the emulator defines the volume of space that contributes to a sample taken along the radar beam. It is bound in elevation and azimuth by a fixed multiple of the half-power beamwidth. This multiple is chosen based on the number of sidelobes that are desired for simulation in the antenna pattern. The pulse is bound in range by the specified pulse length. This volume of space is subdivided into individual pulse elements defined in angular coordinates such that, at the maximum range from the radar, the dimensions of each pulse element are $10 \%$ smaller than the model grid spacing at that range. While there is flexibility in how many subdivisions are made in the pulse element, having too many increases the computation requirements without changing the results. Subdividing the pulse volume into subelements that become larger than the grid spacing at the maximum range of the radar will result in undersampling of the model input fields and will change the emulator output.

Each pulse element is assigned values of extinction cross section, backscattering cross section, and radial velocity that correspond to the grid point nearest to the element's location in space. Nearest-neighbor sampling is chosen over interpolation to improve the computational efficiency of the emulator. Because the pulse elements are generally much smaller than the grid cells, this sampling method provides sufficient accuracy.

The radial velocity is calculated by the projection of the total wind velocity vector onto the radar beam:

$$
V_{r}=(u \sin \theta+v \cos \theta) \cos \varphi+\left(w+w_{t}\right) \sin \varphi,
$$

where $V_{r}$ is the radial velocity, $u$ is the $x$ component of the wind, $v$ is the $y$ component of the wind, $w$ is the $z$ component of the wind, $w_{t}$ is the average terminal fall speed for the hydrometeors, $\theta$ is the azimuth angle measured clockwise from north, and $\varphi$ is the elevation angle. The average hydrometeor terminal fall speed for the grid box is calculated as a backscatter cross-section weighted average given by 


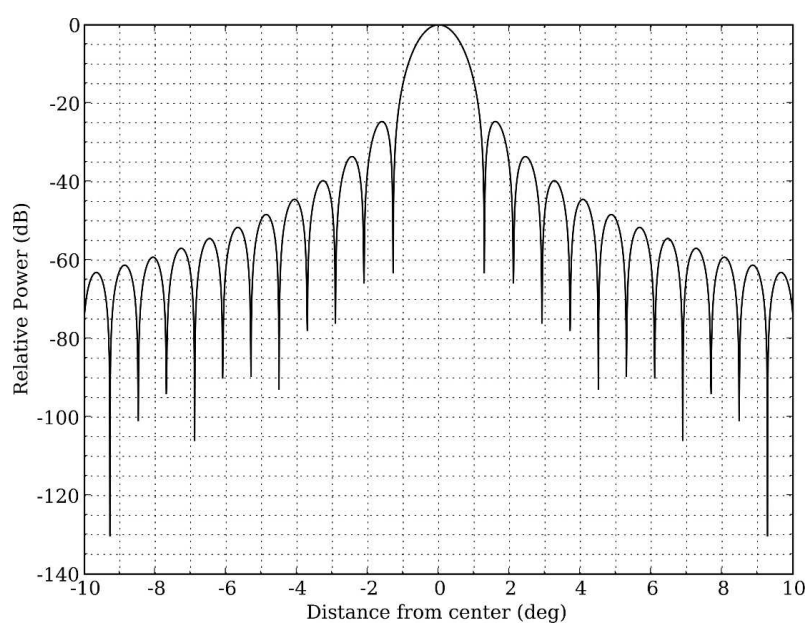

FIG. 1. Emulator antenna pattern for a $1^{\circ}$ half-power beamwidth radar.

$$
w_{t}=\eta^{-1}\left(\frac{\rho_{0}}{\rho}\right)^{0.5} \int \sigma_{b}(D) V_{t}(D) N(D) d D,
$$

where $\eta$ is the total grid reflectivity, $\rho$ is the air density of the grid box, $\rho_{0}$ is the reference density, $N(D)$ is the drop size distribution, and $V_{t}(D)$ is the terminal fall speed as a function of diameter, which is calculated using the fitted relationship of Brandes et al. (2002):

$$
\begin{aligned}
V_{t}(D)= & -0.1021+4.932 D-0.9551 D^{2}+0.07934 D^{3} \\
& -0.002362 D^{4}
\end{aligned}
$$

where $V_{t}$ is in meters per second and $D$ is in millimeters. The weighting by backscatter cross section makes the terminal fall speed more representative of the velocity seen by the radar than a simple mass-weighted average.

The pulse itself is propagated through the numerical grid using a ray-tracing technique. For each range gate, the height of each pulse element is determined separately by taking into account the atmospheric index of refraction experienced by that particular ray element. This allows for differential propagation across the radar beam. The change in the height above ground $\Delta h$ and change in range from the radar (along the surface of the earth) $\Delta r$ can be calculated from the incremental change in range along the path $\Delta s$ as

$$
\Delta h=\left[h^{2}+\Delta s^{2}+2 h \Delta s\left(1-\frac{C^{2}}{n^{2} h^{2}}\right)^{(1 / 2)}\right]^{(1 / 2)},
$$

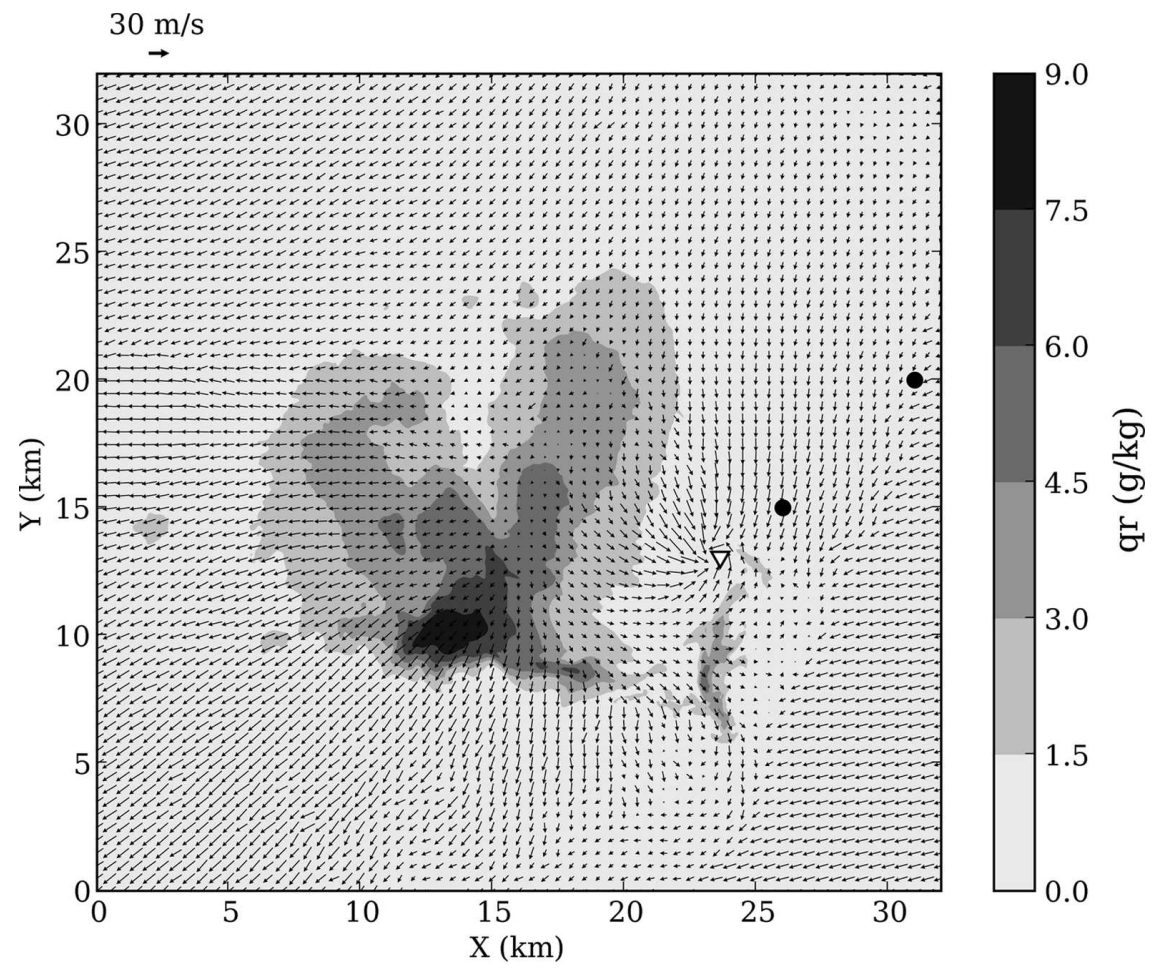

FIG. 2. Model rainwater mixing ratio $\left(q_{r}\right)$ and vector velocity fields at $13500 \mathrm{~s}$ into the simulation and 20-m height. The black dots represent radar locations at ranges of 3 and $10 \mathrm{~km}$. The inverted triangle represents the location of the tornado. 
TABLE 2. Configuration parameters for each experiment.

\begin{tabular}{|c|c|c|c|c|c|c|c|c|c|c|}
\hline Expt & $\begin{array}{c}\lambda \\
(\mathrm{cm})\end{array}$ & $\begin{array}{c}\text { Beamwidth } \\
\left({ }^{\circ}\right)\end{array}$ & PRF (Hz) & $\begin{array}{c}\text { Pulse } \\
\text { length }(\mu \mathrm{s})\end{array}$ & $\begin{array}{l}\text { Rotation } \\
\text { rate }\left({ }^{\circ} \mathrm{s}^{-1}\right)\end{array}$ & $\begin{array}{c}\text { Pulses } \\
\text { per radial }\end{array}$ & $\begin{array}{c}\text { Gate } \\
\text { length }(\mathrm{m})\end{array}$ & $\begin{array}{c}\Delta \mathrm{Az} \\
\left(^{\circ}\right)\end{array}$ & $\begin{array}{l}V_{\mathrm{NYO}} \\
\left(\mathrm{m} \mathrm{s}^{-1}\right)\end{array}$ & $\begin{array}{c}R_{a} \\
(\mathrm{~km})\end{array}$ \\
\hline CNTL & 10 & 1 & 1500 & 1.5 & 20 & 75 & 250 & 1.0 & 37.50 & 100 \\
\hline EXP2 & 10 & 1 & 1500 & 1.5 & 15 & 50 & 250 & 0.5 & 37.50 & 100 \\
\hline EXP3 & 10 & 1 & 1500 & .75 & 20 & 75 & 125 & 1.0 & 37.50 & 100 \\
\hline EXP4 & 10 & 1 & 1500 & 1.5 & 20 & 75 & 250 & 1.0 & 37.50 & 100 \\
\hline EXP5 & 10 & 1 & 1000 & 1.5 & 20 & 50 & 250 & 1.0 & 25.00 & 150 \\
\hline EXP6 & 10 & 2 & 1500 & 1.5 & 20 & 75 & 250 & 1.0 & 37.50 & 100 \\
\hline EXP7 & 10 & 1 & 1500 & 1.5 & 20 & 75 & 250 & 1.0 & 37.50 & 100 \\
\hline EXP8 & 3 & 1 & 1500 & 1.5 & 20 & 75 & 250 & 1.0 & 11.25 & 100 \\
\hline
\end{tabular}

$$
\begin{aligned}
\Delta r & =a \sin ^{-1}\left[\frac{C \Delta s}{n h(h+\Delta h)}\right], \\
C & =n_{0} a \cos \varphi,
\end{aligned}
$$

where $a$ is the radius of the earth, $h$ is the previous height of the element above ground, $n$ is the index of refraction at height $h, n_{0}$ is the index of refraction at the radar, and $\varphi$ is the initial elevation angle of the element (Doviak and Zrnic 1993). The index of refraction is calculated from the model temperature $T$, water vapor pressure $e$, and air pressure $p$, using the relation provided by Bean and Dutton (1966):

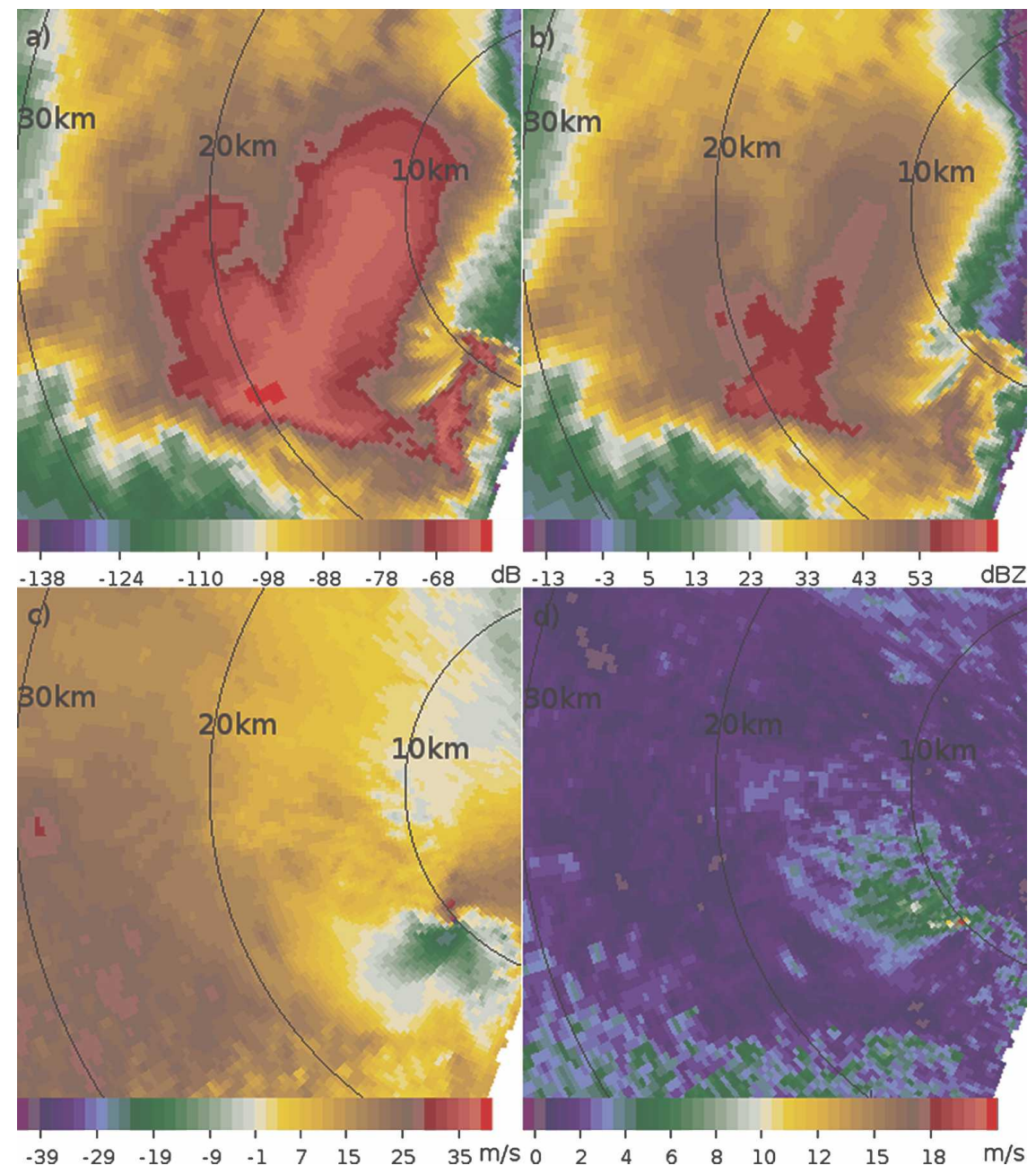

FIG. 3. PPIs of (a) returned power (dB relative to $1 \mathrm{~W}$ ), (b) equivalent reflectivity factor $\left(Z_{e}\right),(\mathrm{c})$ Doppler velocity, and (d) spectrum width for control experiment CNTL. 

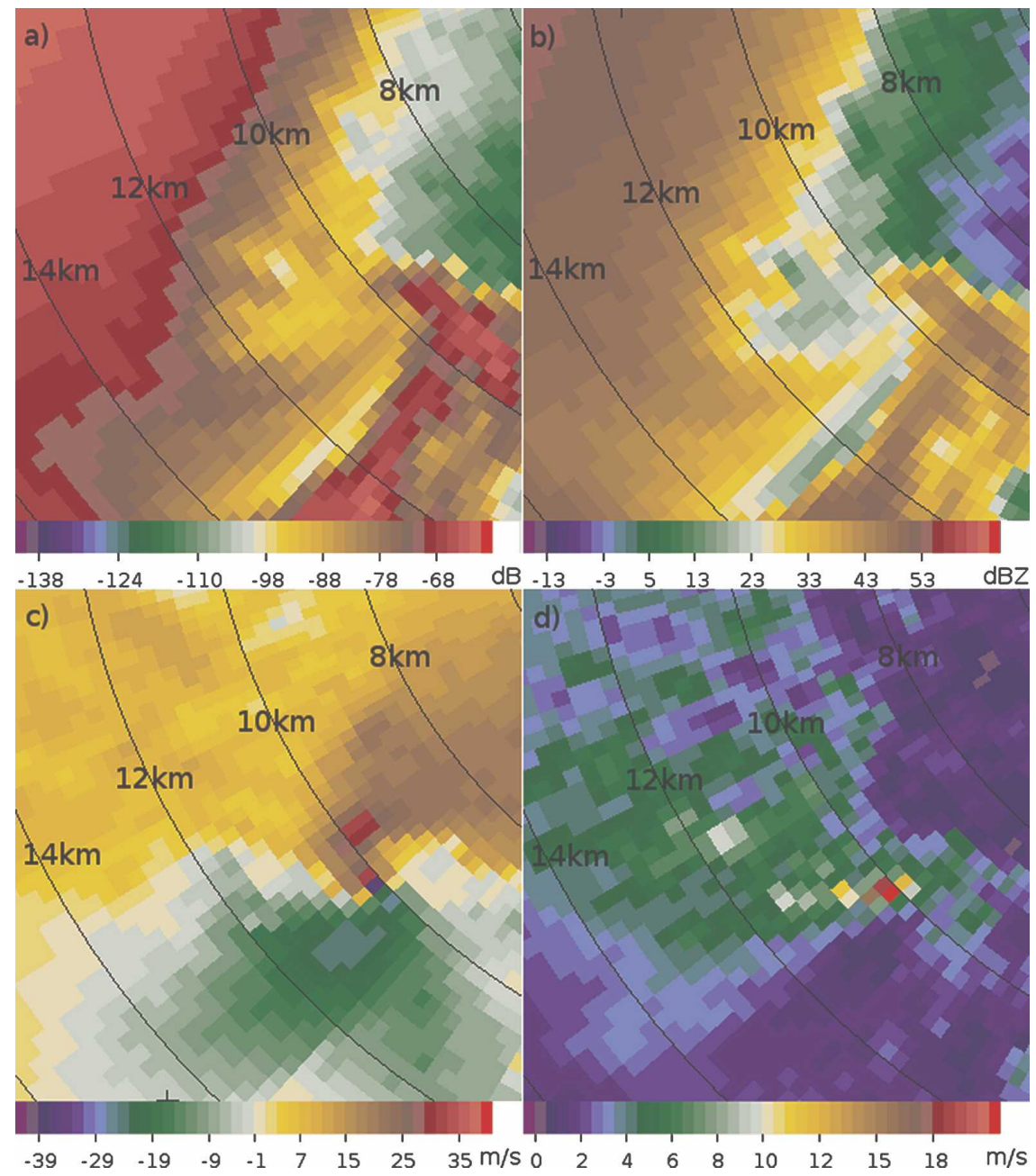

FIG. 4. As in Fig. 3, but magnified to show more details in the region of mesocyclone and tornado.

$$
n=\left(\frac{C_{d} p}{T}+\frac{C_{w 1} e}{T}+\frac{C_{w 2} e}{T^{2}}\right) \times 10^{-6}+1,
$$

where $C_{d}, C_{w 1}$, and $C_{w 2}$, have values of $0.776 \mathrm{~K} \mathrm{~Pa}^{-1}$, $0.716 \mathrm{~K} \mathrm{~Pa}^{-1}$, and $3.7 \times 10^{3} \mathrm{~K}^{2} \mathrm{~Pa}^{-1}$, respectively. The element's range from the radar along the surface of the earth is then converted to standard two-dimensional Cartesian coordinates, which are used to determine the location of the element on the model grid.

The pulse volume is allowed to propagate through the environment as far as twice the unambiguous range $R_{a}$,

$$
R_{a}=\frac{c T_{s}}{2}
$$

where $T_{s}$ is the PRT, and $c$ is the speed of light. Allowing the pulse to propagate $2 R_{a}$ from the radar means that after one PRT from the time the radar is started there are two pulses propagating through the model field at any given instant. Thus, when a sample is taken, the returns from both pulses are assigned to the gate, producing the effects of range aliasing. Range aliasing can be disabled if desired.

\section{d. The calculation of returned power}

The entire pulse volume is stepped forward in range while keeping track of the total extinction cross section along the path. This running total is kept for each pulse element, which allows for the calculation of differential attenuation across the pulse. As the pulse is propagated through the model grid of the simulated atmosphere, it is periodically sampled at an interval in range dictated by the specified gate spacing. This allows for the gate and pulse lengths to be independent. When a pulse sample is taken, three values are calculated: power, power-weighted average radial velocity, and power- 

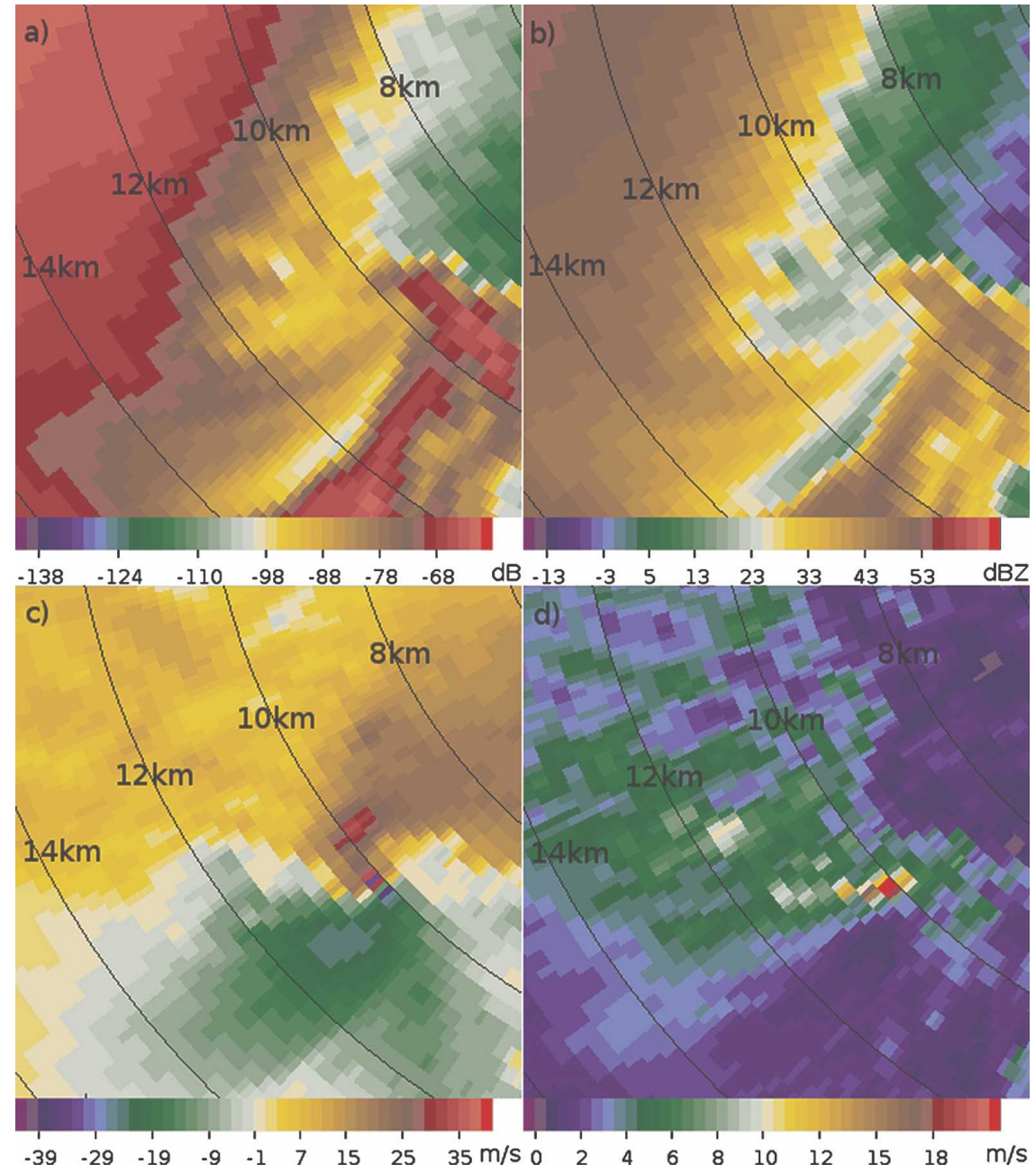

FIG. 5. As in Fig. 4, but for experiment EXP2, showing the effects of azimuthal oversampling.

weighted radial velocity variance. The power weightings are performed over all of the pulse elements as follows:

$$
\begin{gathered}
\bar{V}_{r}=\frac{\sum_{i} P_{i} V_{i}}{\sum_{i} P_{i}}, \\
\sigma_{\mathrm{vr}}=\frac{\sum_{i} P_{i} V_{i}^{2}}{\sum_{i} P_{i}}-\bar{V}_{r}^{2},
\end{gathered}
$$

where $\bar{V}_{r}$ is the power-weighted average radial velocity, $\sigma_{\mathrm{vr}}$ is the power-weighted variance of radial velocities, $P_{i}$ is the power for a particular pulse element, and $V_{i}$ is the radial velocity for a particular pulse element. The estimate of variance here, $\sigma_{\mathrm{vr}}$, is not unbiased but is chosen to simplify the computations. Since values from thousands of pulse elements are used in the calculation, the difference between biased and unbiased estimates is negligible.

As given in Doviak and Zrnic (1993), the power $P$ for a sample taken at range $r_{0}$ is given by

$$
P\left(r_{0}\right)=\iiint \eta(r) I\left(r_{0}, r\right) d V,
$$

where

$$
\begin{aligned}
I\left(r_{0}, r\right) & =\frac{P_{t} g^{2} \lambda^{2} f^{4}(\theta, \varphi)\left|W\left(r_{0}, r\right)\right|^{2}}{(4 \pi)^{3} l^{2}(r) r^{4}}, \\
d V & =r^{2} d r \sin \varphi d \varphi d \theta,
\end{aligned}
$$

and $P_{t}$ is the transmitted power, $g$ is the system gain, $\lambda$ is the wavelength, $r$ is range from the radar, $l$ is the 

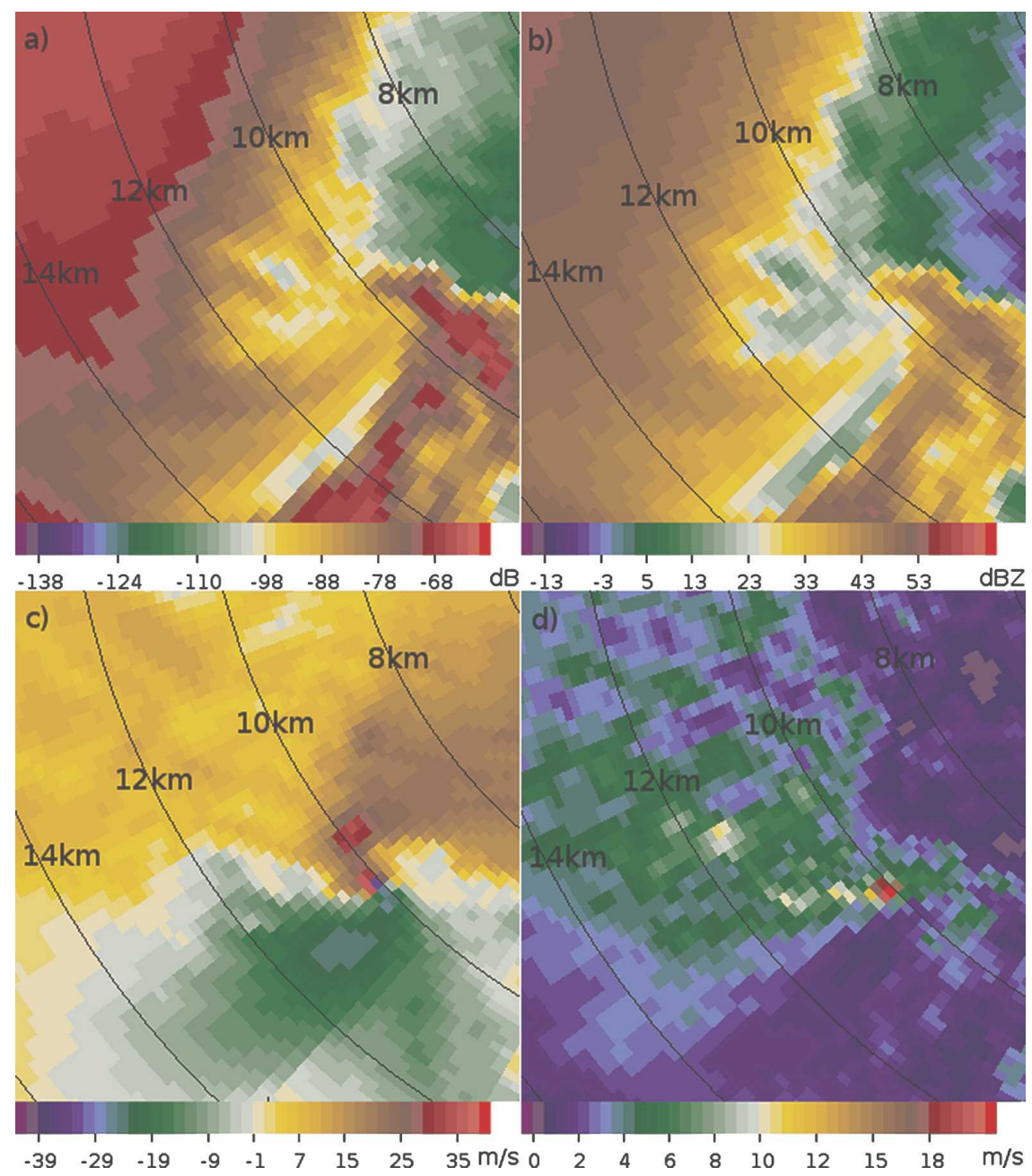

FIG. 6. As in Fig. 4, but for experiment EXP3, showing differences due to a shorter gate spacing and shorter pulse duration.

attenuation factor, $f^{2}$ is the normalized antenna pattern, $\eta$ is the reflectivity (backscattering cross section per unit volume), $\theta$ is the azimuth angle relative to the beam center, $\varphi$ is the elevation angle relative to beam center, and $W$ is the range-weighting function. The emulator approximates this integral with a sum over the finite elements within the pulse volume,

$$
P\left(r_{0}\right)=\frac{P_{t} g^{2} \lambda^{2}}{(4 \pi)^{3}} \sum_{i} \frac{f_{i}^{4} W_{i}^{2} \eta_{i} \Delta V_{i}}{l_{i}^{2} r_{i}^{2}}
$$

where $\Delta V$ is the volume of a pulse element, and all quantities subscripted with $i$ are values for a particular pulse element. The emulator assumes a Gaussian range-weighting function and a normalized antenna pattern with the following form (Doviak and Zrnic 1993):

$$
f^{2}(\theta)=\left[\frac{8 J_{2}\left(\pi D_{a} \sin \theta / \lambda\right)}{\left(\pi D_{a} \sin \theta / \lambda\right)^{2}}\right]^{2}
$$

where $J_{2}$ is the second-order Bessel function of the first kind, $\theta$ is the angular offset from boresight, and $D_{a}$ is the diameter of the antenna, which for (21) above can be calculated from the half-power beamwidth $\theta_{1}$ as

$$
D_{a}=\frac{1.27 \lambda}{\theta_{1}},
$$

where $\lambda$ is the wavelength. Doviak and Zrnic (1993) state that (21) describes the antenna pattern for the first few sidelobes quite well for a parabolic antenna. However, (21) is limited in that it gives sidelobes of a fixed level and location (e.g., Fig. 1), prohibiting configuration of sidelobes with arbitrary magnitude.

\section{e. Moment calculation}

The sampling of model data is repeated for the number of pulses that are to be averaged for a radial of data, as specified by the scanning strategy. Moment data 
(power, Doppler velocity, and Doppler spectrum width) are then generated at each range gate along the radial. Power is calculated as the average of all power samples for the specified number of pulses at that range gate. Note that this is the expected mean power that an actual weather radar would produce if random power fluctuations were successfully removed by the pulse averaging and the radar system had no noise.

Radial velocity is calculated as the power-weighted average of all velocity samples (one per pulse) at that range gate. To emulate velocity aliasing, this average is restricted to a value within the Nyquist interval and is given by

$$
V_{a}=V_{r}+2 n V_{\mathrm{NYQ}},
$$

where

$$
\begin{aligned}
& n=0 \text { for }\left|V_{r}\right| \leq V_{\mathrm{NYQ}}, \\
& n=\frac{V_{\mathrm{NYQ}}-V_{r}}{2 V_{\mathrm{NYQ}}}-1 \text { for } V_{r}>V_{\mathrm{NYQ}}, \\
& n=\frac{-V_{\mathrm{NYQ}}-V_{r}}{2 V_{\mathrm{NYQ}}}+1 \text { for } V_{r}<-V_{\mathrm{NYQ}},
\end{aligned}
$$

where $V_{a}$ is the aliased velocity value; $V_{r}$ is the original (unaliased) radial velocity; $V_{\mathrm{NYQ}}$ is the Nyquist (or

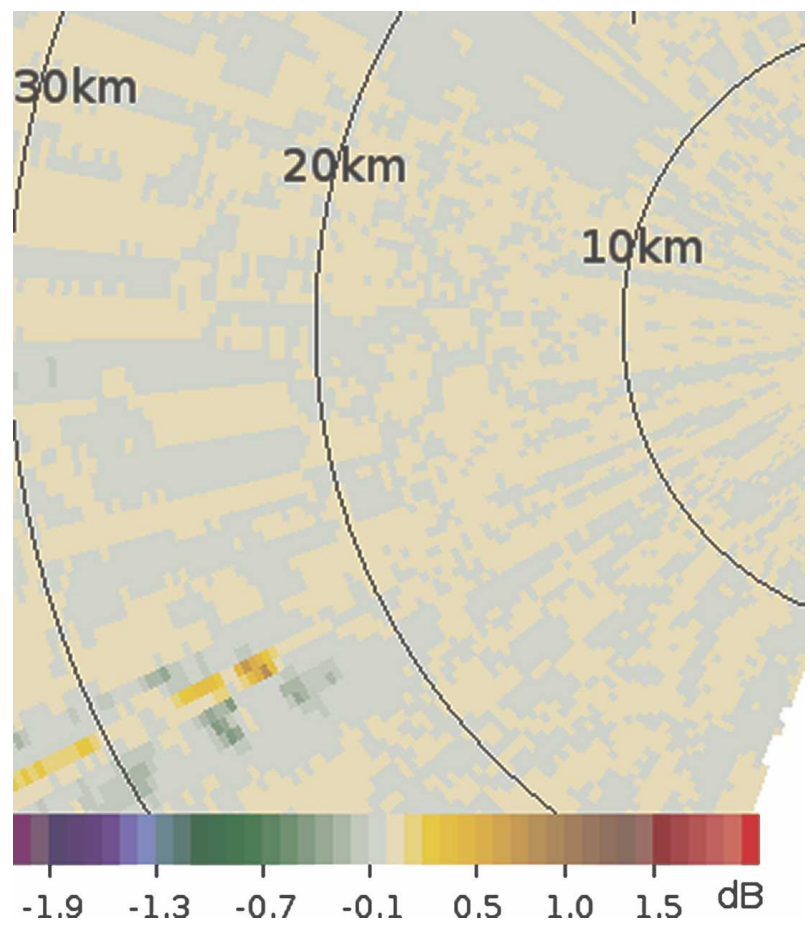

FIG. 7. PPI of returned power difference between CNTL and EXP4 (CNTL subtracted from EXP4), showing overall minimal differences due to sidelobes. Areas where EXP4 has less returned power are due to numeric instability in the computations. aliasing) velocity; and $n$, an integer, is the number of Nyquist intervals by which the $V_{a}$ differs from $V_{r}$. One advantage of emulated data is that the unaliased Doppler velocity is known. Spectrum width is calculated as the power-weighted average of the variance for each sample, which is the variance of all velocity values within the pulse. Initial attempts at emulating spectrum width used only the variance of the individual velocity samples that were themselves an average over the entire pulse. That approach produced unreasonably low spectrum width. By taking into account the variance of all velocity values within all pulses, the spectrum width takes into account the effect of antenna rotation and wind shear across the radar beam. However, we have neglected subgrid-scale atmospheric turbulence. Moreover, since the emulator does not generate a true power spectrum at each range gate, the emulated spectrum width does not take into account a limited Nyquist interval or the pulse-to-pulse variability associated with random phase changes from scatterers moving relative to the transmitted wavelength. In addition to the three moments above, equivalent reflectivity factor $\left(Z_{e}\right)$ is calculated from the average power $P_{r}$, using

$$
Z_{e}=\frac{2^{10}(\ln 2) \lambda^{2} r^{2} \overline{P_{r}}}{\pi^{3} P_{t} g^{2} \theta_{1}^{2} c \tau\left|K_{w}\right|^{2}},
$$

where $\tau$ is the pulse duration.

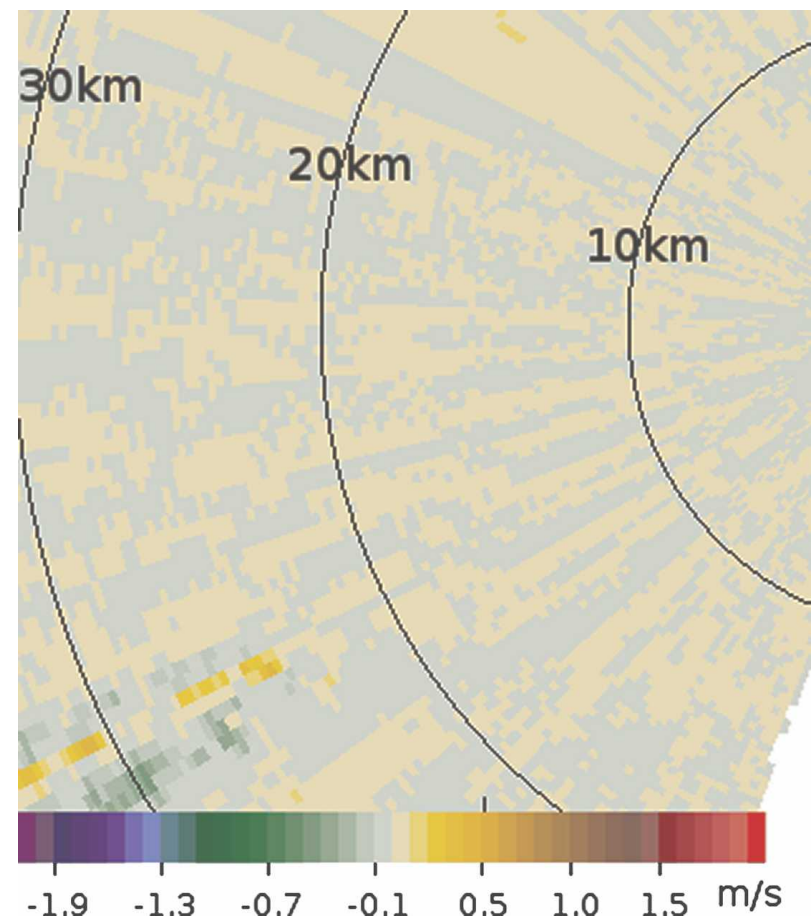

FIG. 8. As in Fig. 7, but for Doppler velocity difference, showing the small impact of sidelobes on measured Doppler velocity. 

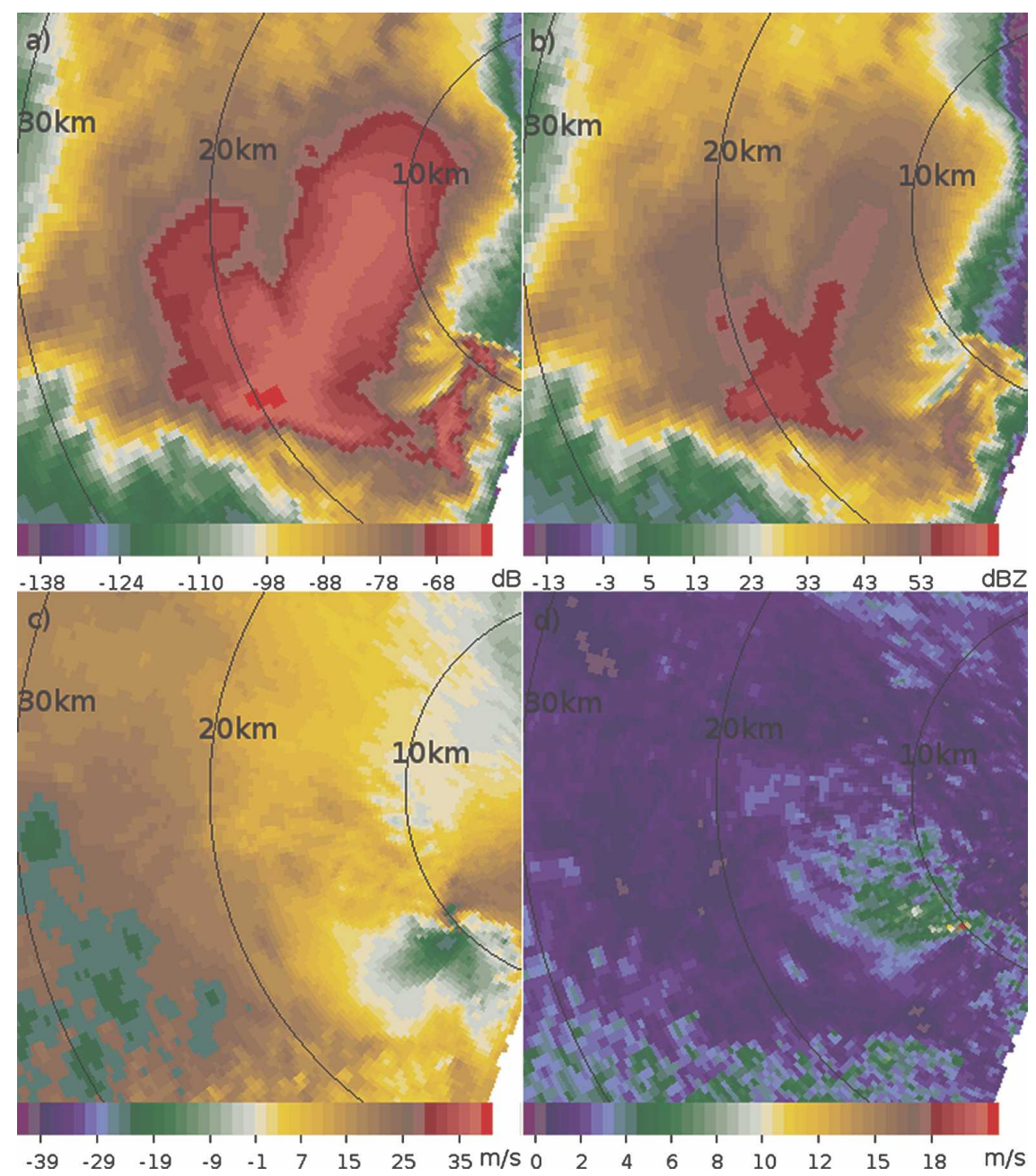

FIG. 9. As in Fig. 3, but for experiment EXP5, showing the impacts of reducing the PRF.

\section{Demonstration of emulator capabilities}

Emulated data were generated for different radar characteristics to illustrate the emulator's capabilities and to demonstrate the impact of radar design on data quality. The input is from a numerical simulation of a supercell thunderstorm produced using the Advanced Regional Prediction System (ARPS; Xue et al. 2000, 2001). The ARPS is a fully compressible and nonhydrostatic prediction model, and its prognostic state variables include wind components $u, v, w$; potential temperature $\theta$; pressure $p$; the mixing ratios for water vapor $q_{v}$; cloud water $q_{c}$; rainwater $q_{r}$; cloud ice $q_{i}$; snow $q_{s}$; and hail $q_{h}$; plus the turbulent kinetic energy used by the 1.5-order subgrid-scale turbulent closure scheme.

For the current simulation, only liquid-phase Kessler (1969) microphysics is used. The simulation had a horizontal grid spacing of $50 \mathrm{~m}$ over a $48 \mathrm{~km}$ by $48 \mathrm{~km}$ domain and a vertically stretched grid that goes from the surface to $16 \mathrm{~km}$. The stretching is specified by a hyperbolic tangent function, having a minimum spacing of $20 \mathrm{~m}$ at the surface, 380-m spacing at the top of the model, and a mean spacing of $200 \mathrm{~m}$ (Xue et al. 1995). The model thunderstorm was initiated by a thermal bubble in a horizontally homogeneous environment defined by the 20 May 1977 Del City, Oklahoma, supercell sounding reported in Ray et al. (1981). Detailed analysis of the simulated storm is unimportant here as the simulation serves only as input to the emulator. Furthermore, only a single time step taken during the most intense portion of the tornadic stage of the simulated storm is used. The impact of storm evolution on radar-derived storm structure will be the subject of future studies.

Figure 2 shows the rainwater mixing ratio and stormrelative velocity at the surface at 13500 s into the simulation, the time used to produce the emulated data. An intense supercell thunderstorm with characteristic v- 



FIG. 10. As in Fig. 4, but for experiment EXP6, showing the impacts of changing the half-power beamwidth.

notch and hook (Lemon and Doswell 1979) in the rainwater field is evident. The simulated tornado vortex is about $200 \mathrm{~m}$ in diameter, with maximum winds of about $75 \mathrm{~m} \mathrm{~s}^{-1}$.

Table 2 lists the parameters used to define the radar and scanning strategy in each of the experiments discussed below.

\section{a. Control experiment for $S$-band radar with $1^{\circ}$ beamwidth}

The control experiment (CNTL), against which other radar configurations are compared, assumes characteristics similar to those of the U.S. National Weather Service WSR-88D (Crum and Alberty 1993) operational weather radars. The WSR-88Ds operate at a nominal wavelength of $10 \mathrm{~cm}$ with a peak power of $750 \mathrm{~kW}$ and have a nominal half-power beamwidth of $1^{\circ}$. The cur- rent operational scanning strategy uses a $1^{\circ}$ azimuthal sampling interval (which is close to the beamwidth), with range gates spaced $250 \mathrm{~m}$ apart. For these experiments, the radar is located $20 \mathrm{~km}$ north of the southern edge of the model domain and $31 \mathrm{~km}$ east of the western edge of the domain, or about $10 \mathrm{~km}$ northeast of the center of the tornadic circulation. Except where noted, the antenna has no sidelobes but is restricted to the area between the first nulls in the antenna pattern, keeping the full main lobe of the antenna. The width of this region for a $1^{\circ}$ half-power beamwidth antenna is approximately $3^{\circ}$. PPIs of emulated power, equivalent reflectivity factor $Z_{e}$, Doppler radial velocity, and spectrum width (Fig. 3) for this experiment show the familiar reflectivity structure (Fig. 3b) of a supercell thunderstorm, with a pronounced hook echo (magnified in Fig. 4). A pronounced mesocyclone circulation (Figs. $3 c, 4 c)$, with a small region of high gate-to-gate shear 


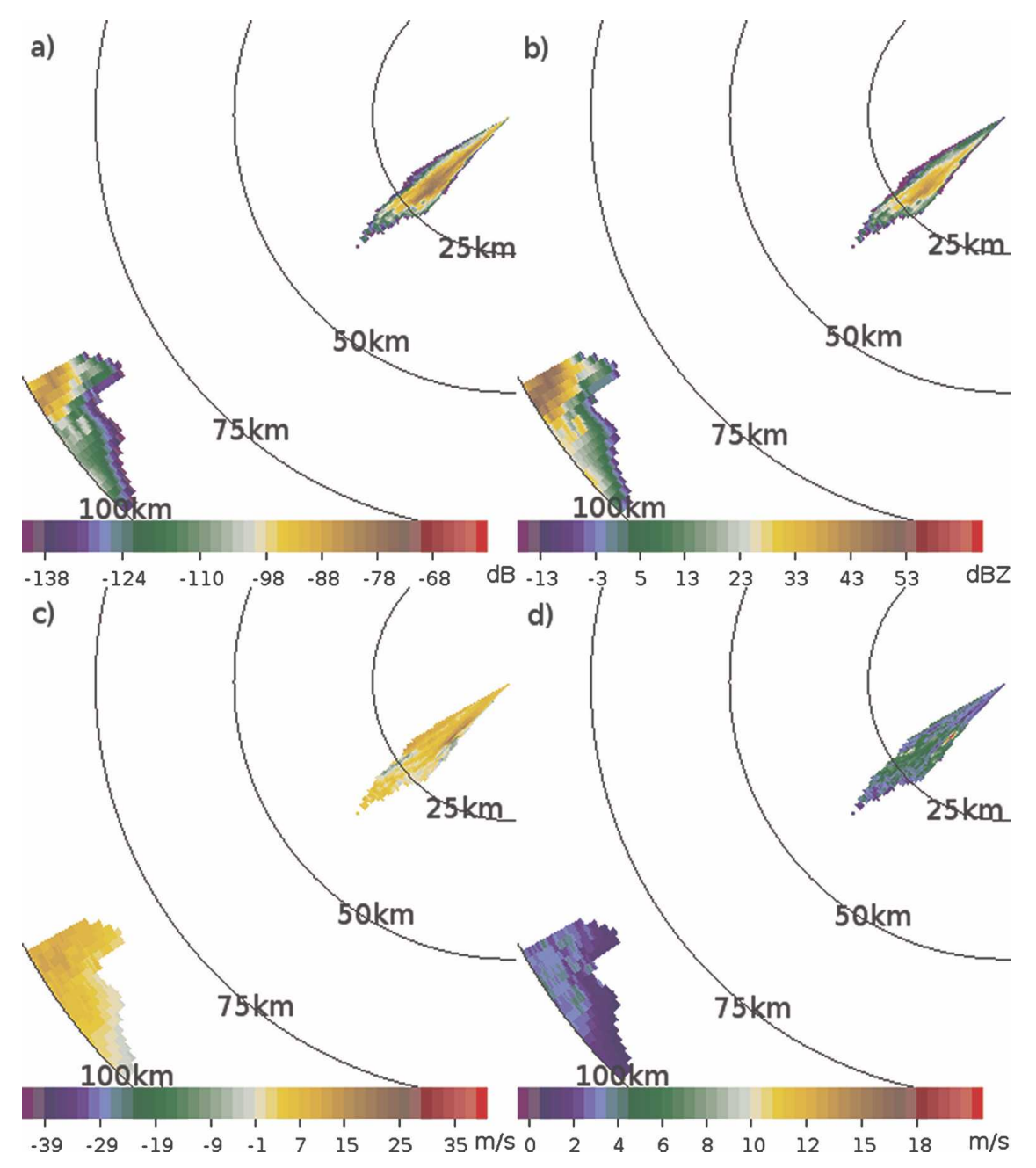

FIG. 11. As in Fig. 3, but for experiment EXP7, showing second-trip echoes. The scale has been changed to allow both first- and second-trip echoes to be shown.

corresponding to the tornado, was found at the tip of the hook echo. The spectrum width (Figs. 3d, 4d) was relatively low, 1-3 $\mathrm{m} \mathrm{s}^{-1}$, for most of the storm. However, the spectrum width was higher $\left(>4 \mathrm{~m} \mathrm{~s}^{-1}\right)$ in the region of the mesocyclone, reaching a maximum of $\sim 20$ $\mathrm{m} \mathrm{s}^{-1}$ around the tornado. It should also be noted that the Doppler velocity field for this experiment exhibits almost no aliasing, except for a single velocity gate, because of the high Nyquist velocity $\left(37.5 \mathrm{~m} \mathrm{~s}^{-1}\right)$ of the CNTL run. Even at this range, the $75 \mathrm{~m} \mathrm{~s}^{-1}$ flow in the simulated tornado was significantly reduced by averaging across the $1^{\circ}$ half-power beamwidth. Similar reduction in vortex strength by beam averaging was noted by Wood and Brown (1997).

\section{b. Oversampling in azimuth}

Experiment EXP2 (Table 2) is identical to CNTL, except that fewer pulses (50 instead of 75) are used to generate a radial, and the antenna is rotated at $15^{\circ} \mathrm{s}^{-1}$ instead of $20^{\circ} \mathrm{s}^{-1}$, yielding data that are azimuthally oversampled relative to the beamwidth. This difference in azimuthal sampling resulted in differences in the observed structure of the storm, especially in the tornadic region (Fig. 5). Overall, azimuthal oversampling yielded finer-scale structure of the storm (cf. Fig. 4). Of particular interest are the velocity measurements around the tornado; the oversampled data produce higher inbound and outbound velocities than the beammatched CNTL case. These increases in velocity values, though minimal, are due to the decreased region that is averaged, which allows the peak velocity values in the tornado to contribute more to the sampled Doppler velocity value. Wood et al. (2001) and Brown et al. (2002) report a similar result for an idealized Rankine vortex flow and a simpler radar emulator. Using time series data taken from a WSR-88D during a tornadic 

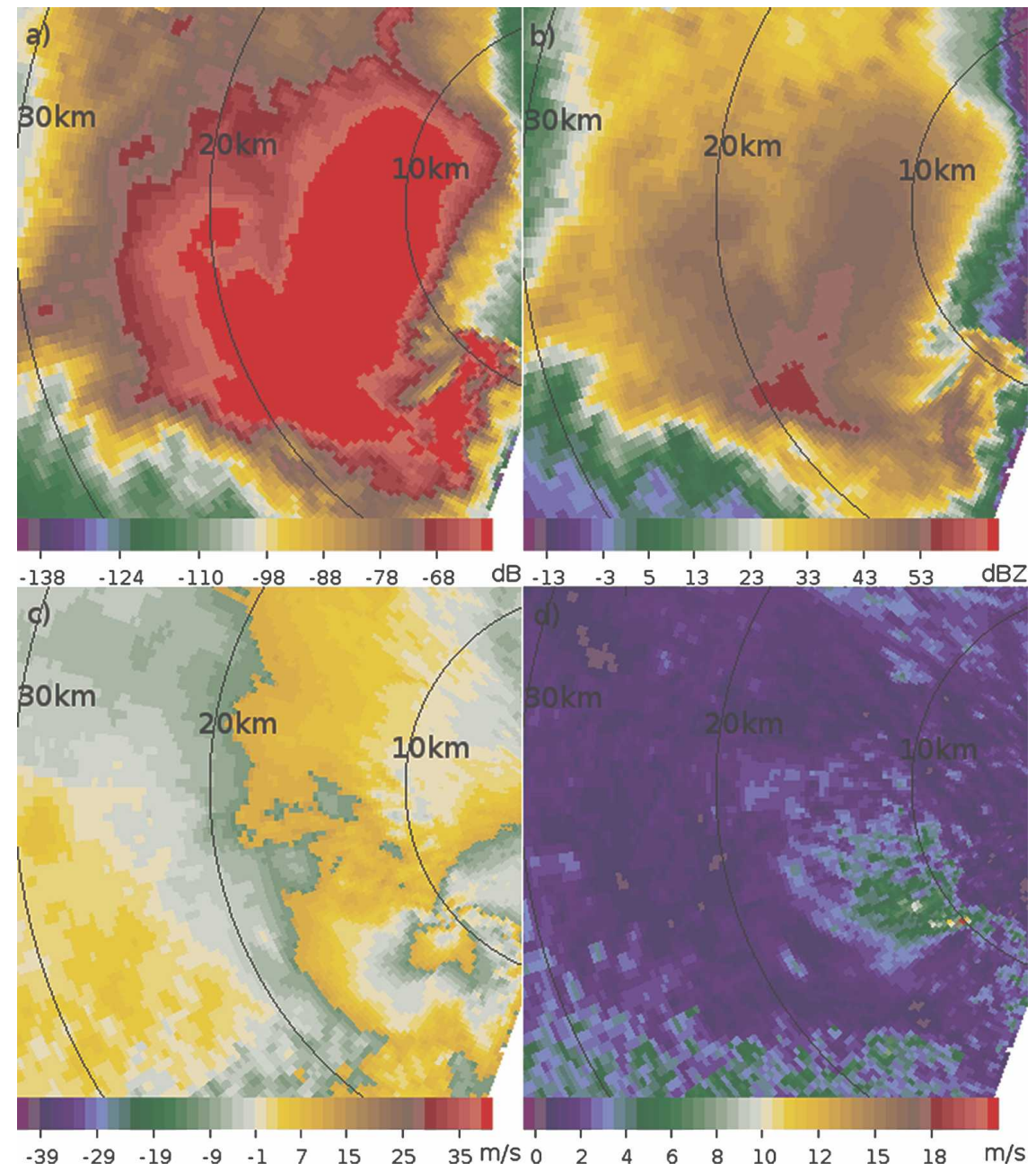

FIG. 12. As in Fig. 3, but for experiment EXP8, highlighting the storm structure observed at $\mathrm{X}$ band.

supercell, Wood et al. (2001) demonstrated that oversampling by a factor of 2 increased the observed mesocyclone strength by $10 \%-50 \%$ relative to standard azimuthal sampling for one-third of the mesocyclones detected.

\section{c. Effects of gate length}

Experiment EXP3 (Fig. 6) differs from CNTL by using a smaller gate length, $125 \mathrm{~m}$ instead of $250 \mathrm{~m}$, and a correspondingly smaller pulse length, $0.75 \mu$ s instead of $1.5 \mu \mathrm{s}$, resulting in a higher range resolution. As in experiment EXP2, the shorter sampling interval in the radial direction results in the elucidation of finer-scale flow, especially in the region of the tornado. Due to the smaller region sampled (and averaged) in range, higher inbound and outbound velocity values are obtained, though not as high as the azimuthally oversampled case. The latter is expected because azimuthal oversampling is more effective in capturing the extreme values of inbound and outbound velocities in quasiaxisymmetric flow. This fact was also the motivation of the work of Xue et al. (2007).

\section{d. Effects of sidelobes}

Experiment EXP4 repeated the CNTL experiment with the pulse expanded to $6^{\circ}$ in azimuth, which included the first two antenna sidelobes. For the antenna pattern used here, the first sidelobe had a one-way gain that was $28 \mathrm{~dB}$ less than the peak of the main lobe. Since ground clutter was not included, this experiment exhibited only minor differences from CNTL (Figs. 7 and 8). Regions with the strongest reflectivity gradients exhibited a few tenths of a decibel change in returned power and a few tenths of a meter per second differ- 


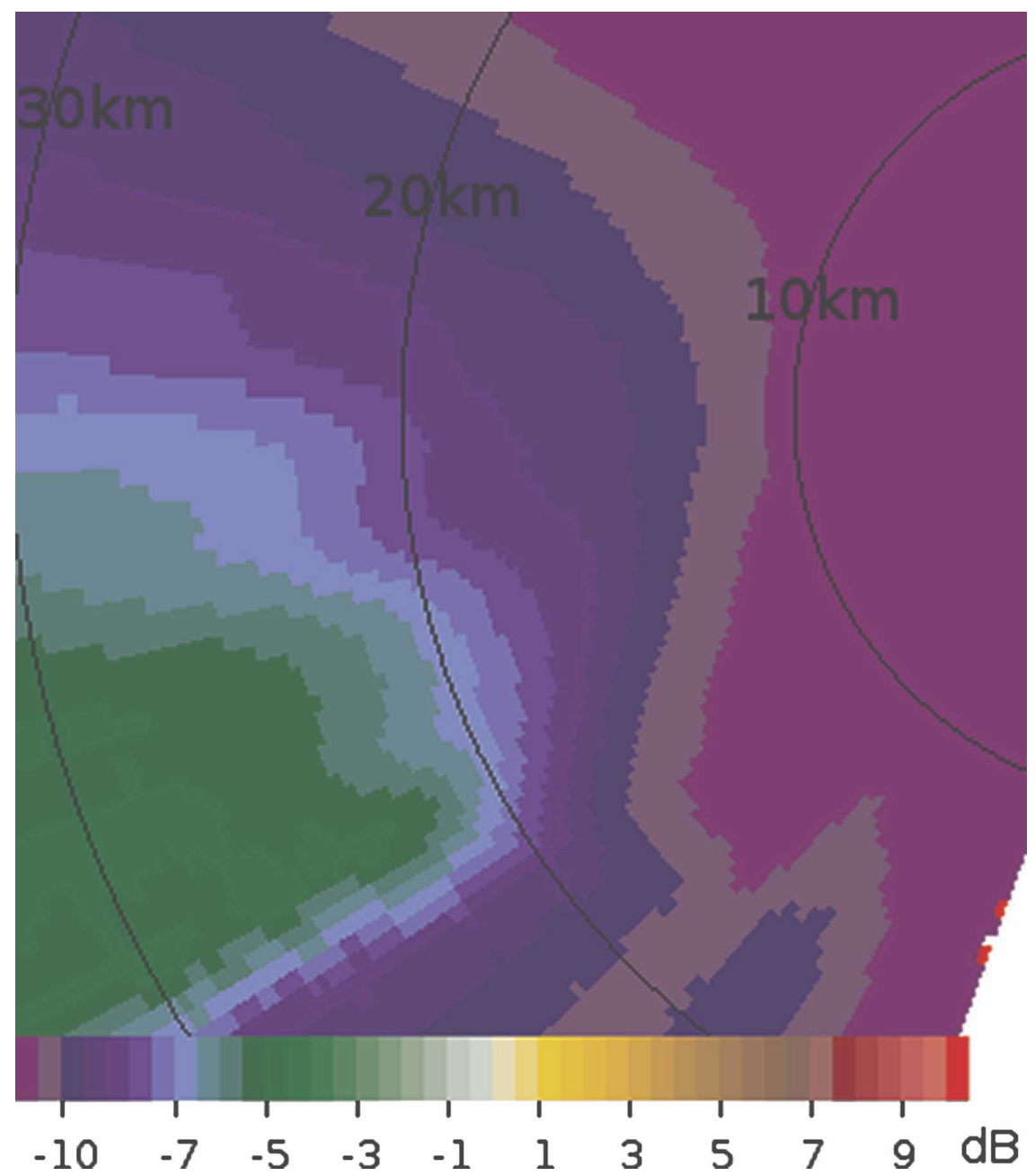

FIG. 13. PPI of returned power difference between CNTL and EXP8, showing clearly the range propagation effect of Rayleigh attenuation at $\mathrm{X}$ band.

ence in wind speed. The area around the tornado had almost no change in diagnosed velocity, suggesting that the WSR-88D velocity measurements in such storms are not strongly affected by sidelobes in the absence of ground clutter.

While the increase in volume contributing to returned power in EXP4 should have led to consistently higher values, there are a few places where less power was found. The lower power results from slight changes in the antenna gain weighting assigned to individual grid elements illuminated by the radar beam between the two runs. In essence, the center of the beam is not located in exactly the same place because of truncation in the numerical calculations of the beam projection through the model grid. These small errors led to places in which the sidelobes' run had lower power than the CNTL run without sidelobes. We speculate that once ground clutter is included the enhanced return from the ground will overwhelm this numerical artifact and re- sult in higher reflectivity uniformly across the radar domain at small elevation angles.

\section{e. Experiment with PRF and effects on velocity aliasing}

In experiment EXP5, the pulse repetition frequency (PRF) was set to $1000 \mathrm{~Hz}$ instead of the $1500 \mathrm{~Hz}$ in

TABLE 3. Configuration parameters for CASA radars.

\begin{tabular}{lcc}
\hline \hline Radar parameter & Matched sampling & Oversampled \\
\hline$\lambda(\mathrm{cm})$ & 3 & 3 \\
Beamwidth $\left({ }^{\circ}\right)$ & 2 & 2 \\
PRF $(\mathrm{Hz})$ & 2000 & 2000 \\
Rotation rate $\left({ }^{\circ} \mathrm{s}^{-1}\right)$ & 40 & 40 \\
Pulses per radial & 100 & 50 \\
Pulse length $(\mu \mathrm{s})$ & 0.5 & 0.5 \\
Gate length $(\mathrm{m})$ & 100 & 100 \\
$\Delta \mathrm{Az}\left({ }^{\circ}\right)$ & 2 & 1 \\
\hline
\end{tabular}


TABLE 4. Calculated tornado parameters for emulated CASA radars.

\begin{tabular}{lcrrr}
\hline \hline $\begin{array}{c}\text { Expt (radar range, } \\
\text { oversampling) }\end{array}$ & $\begin{array}{c}V_{\max } \\
\left(\mathrm{m} \mathrm{s}^{-1}\right)\end{array}$ & $\begin{array}{c}\Delta V \\
\left(\mathrm{~m} \mathrm{~s}^{-1}\right)\end{array}$ & $\begin{array}{c}D \\
(\mathrm{~m})\end{array}$ & $\begin{array}{c}2 \Delta V / D \\
\left(\mathrm{~s}^{-1}\right)\end{array}$ \\
\hline $3 \mathrm{~km}$, matched & 49.1 & 93.3 & 216 & 0.864 \\
$3 \mathrm{~km}$, oversampled & 55.7 & 110.6 & 216 & 1.024 \\
$10 \mathrm{~km}$, matched & 35.2 & 57.6 & 705 & 0.163 \\
$10 \mathrm{~km}$, oversampled & 36.3 & 62.7 & 529 & 0.237 \\
$30 \mathrm{~km}$, matched & 31.8 & 33.4 & 1047 & 0.064 \\
$30 \mathrm{~km}$, oversampled & 32.3 & 42.7 & 1047 & 0.082 \\
$50 \mathrm{~km}$, matched & 27.5 & 29.5 & 1749 & 0.034 \\
$50 \mathrm{~km}$, oversampled & 28.5 & 38.6 & 1749 & 0.044 \\
\hline
\end{tabular}

CNTL, and 50 pulses were used for each radial instead of 75 , to keep the azimuthal sampling interval the same $\left(1^{\circ}\right)$. This changes the Nyquist velocity from $37.5 \mathrm{~m} \mathrm{~s}^{-1}$ for CNTL to $25 \mathrm{~m} \mathrm{~s}^{-1}$ for EXP5. Figure 9c shows a PPI of the Doppler velocity for this case. The reduced Nyquist velocity causes more velocity aliasing, especially in the mesocyclone and tornado region. There are also subtle differences in the $Z_{e}$ (Fig. 9b) and spectrum width (Fig. 9d) fields, which are caused by the change to a lower PRF and using fewer samples to generate a radial of data. This experiment illustrates the advantage of using high PRFs to reduce velocity aliasing. This becomes particularly important at shorter wavelengths, since the Nyquist velocity scales linearly with the transmitted wavelength.

\section{f. Experiment with beamwidth}

In EXP6 the half-power beamwidth was increased from $1^{\circ}$ to $2^{\circ}$ (effectively halving the diameter of the dish) while keeping the azimuthal sampling interval the same. This effectively yields azimuthally oversampled
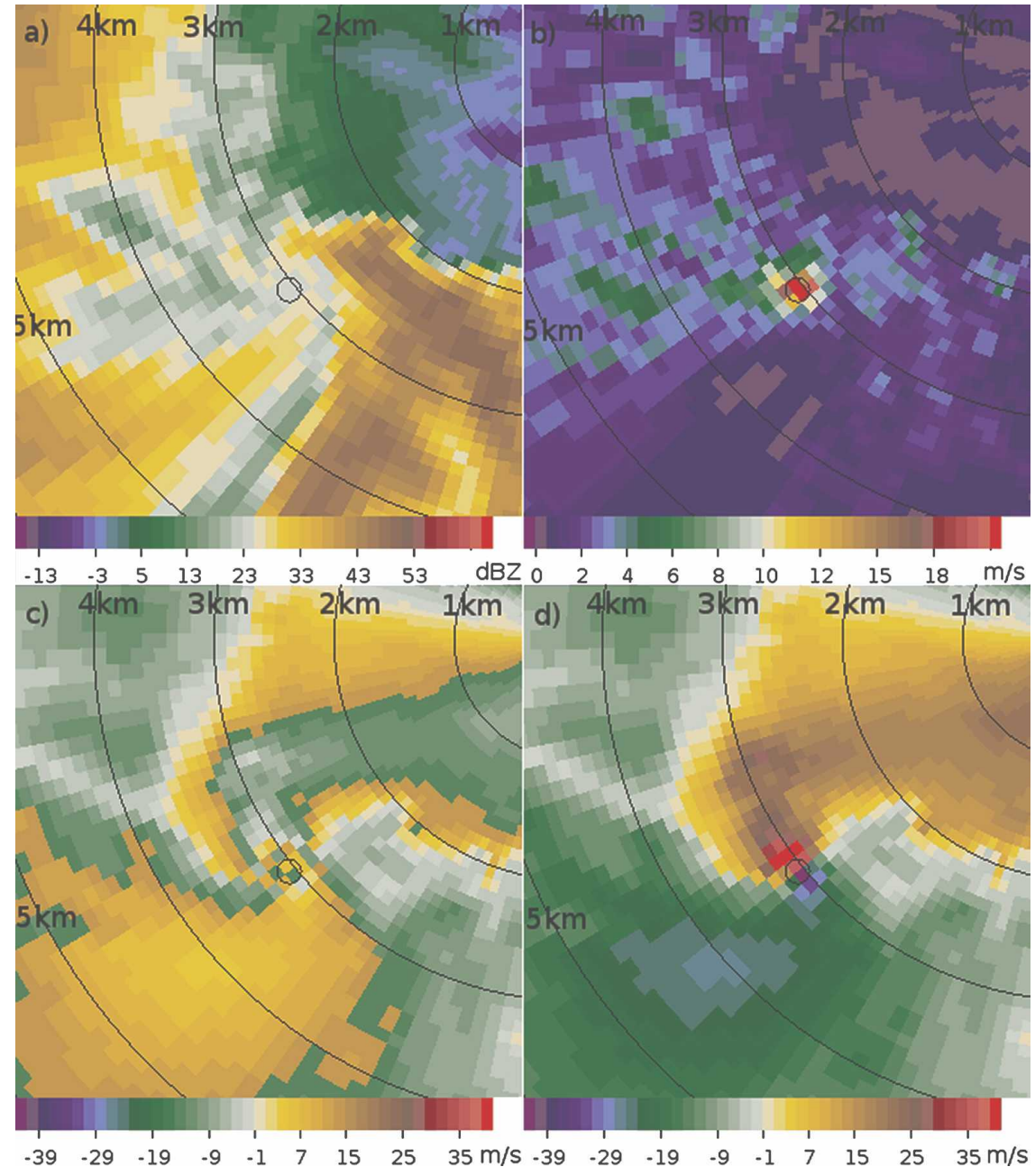

FIG. 14. PPIs of (a) equivalent reflectivity factor, (b) spectrum width, (c) aliased Doppler velocity, and (d) nonaliased Doppler velocity for a radar located $3 \mathrm{~km}$ from the tornado using matched sampling. The black circle indicates the location and size of the tornado in the model. 

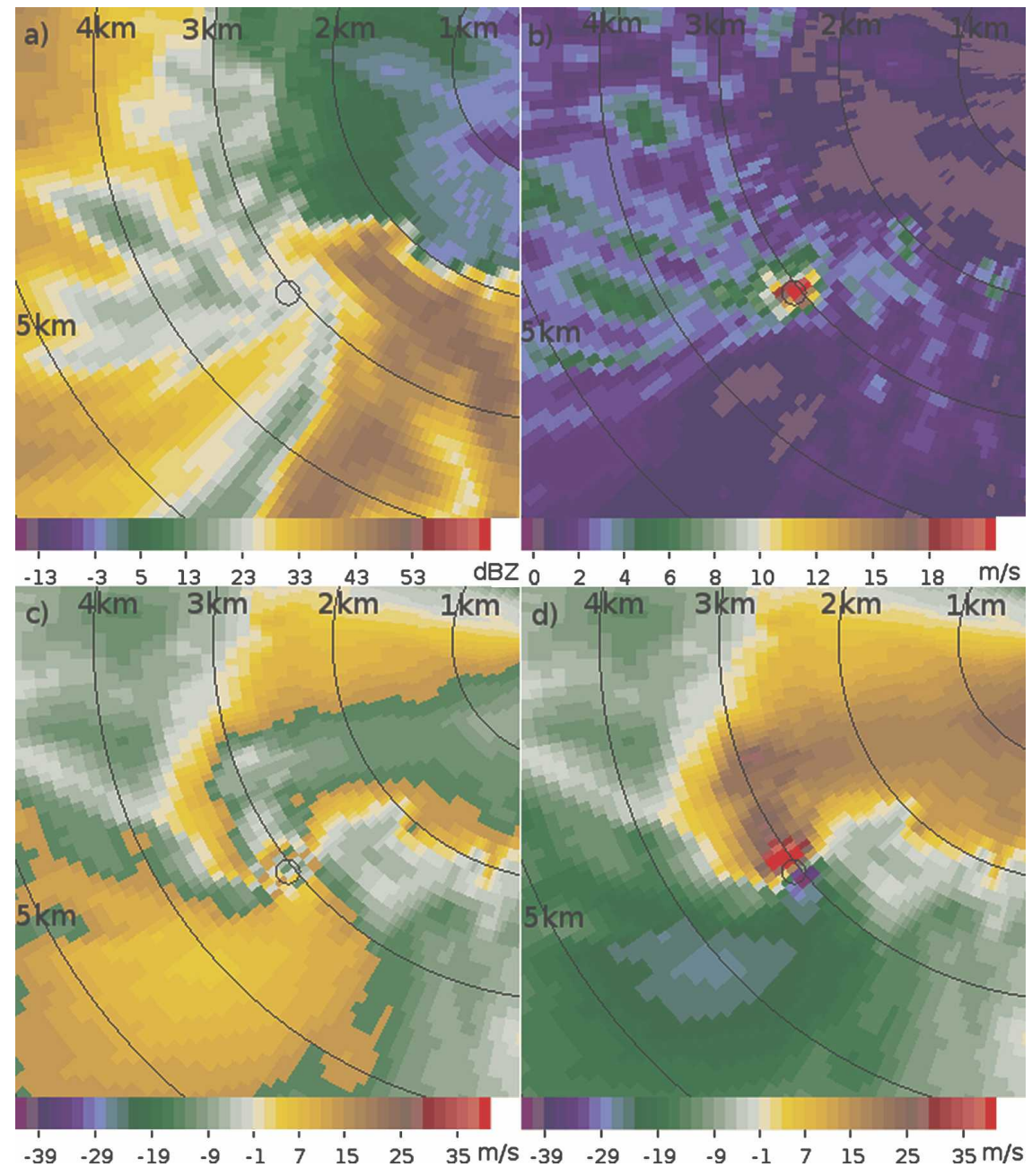

FIG. 15. As in Fig. 14, but for the radar azimuthally oversampling by a factor of 2 .

data, since the $1^{\circ}$ azimuthal sampling interval is smaller than the antenna's half-power beamwidth (Fig. 10). Comparing the data with those from CNTL (Fig. 4), it is clear that the broader beam decreases the peak velocities retrieved by the emulator within the tornado (Fig. 10c). Specifically, the maximum outbound velocity is decreased from 38 to $31 \mathrm{~m} \mathrm{~s}^{-1}$. Changing the halfpower beamwidth also increases the spectrum width in the entire region of mesocyclone, which is a consequence of the larger sampling volumes.

\section{g. Second-trip echoes and range aliasing}

The purpose of EXP7 was to demonstrate the emulator's ability to simulate range aliasing. In this case, the radar is located approximately $100 \mathrm{~km}$ from the storm's mesocyclone. Otherwise, the scanning strategy is the same as CNTL, which had an unambiguous range of $100 \mathrm{~km}$. With this scanning strategy, part of the storm is located beyond the unambiguous range, resulting in second-trip echoes from 0- to $35-\mathrm{km}$ range (Fig. 11). These echoes look very narrow as a result of the fixed angular resolution of the data, which causes distortion since the data are assigned to a much closer range than their actual location. It is important to note that currently the velocities from the second-trip echoes are determined by assuming a fixed-phase transmitter, like a klystron, which makes the Doppler velocities and spectrum widths of the second-trip echoes coherent. Emulation of a random phase transmitter, like a magnetron, could be accomplished by assigning a random value of velocity for each sample of the radar pulse for the second-trip echo.

\section{h. Radar wavelength and effects on attenuation and velocity aliasing}

EXP8 was identical to CNTL, except that the transmitted wavelength of the radar was changed from $10 \mathrm{~cm}$ ( $\mathrm{S}$ band) to $3 \mathrm{~cm}$ (X band). Comparing the returned 

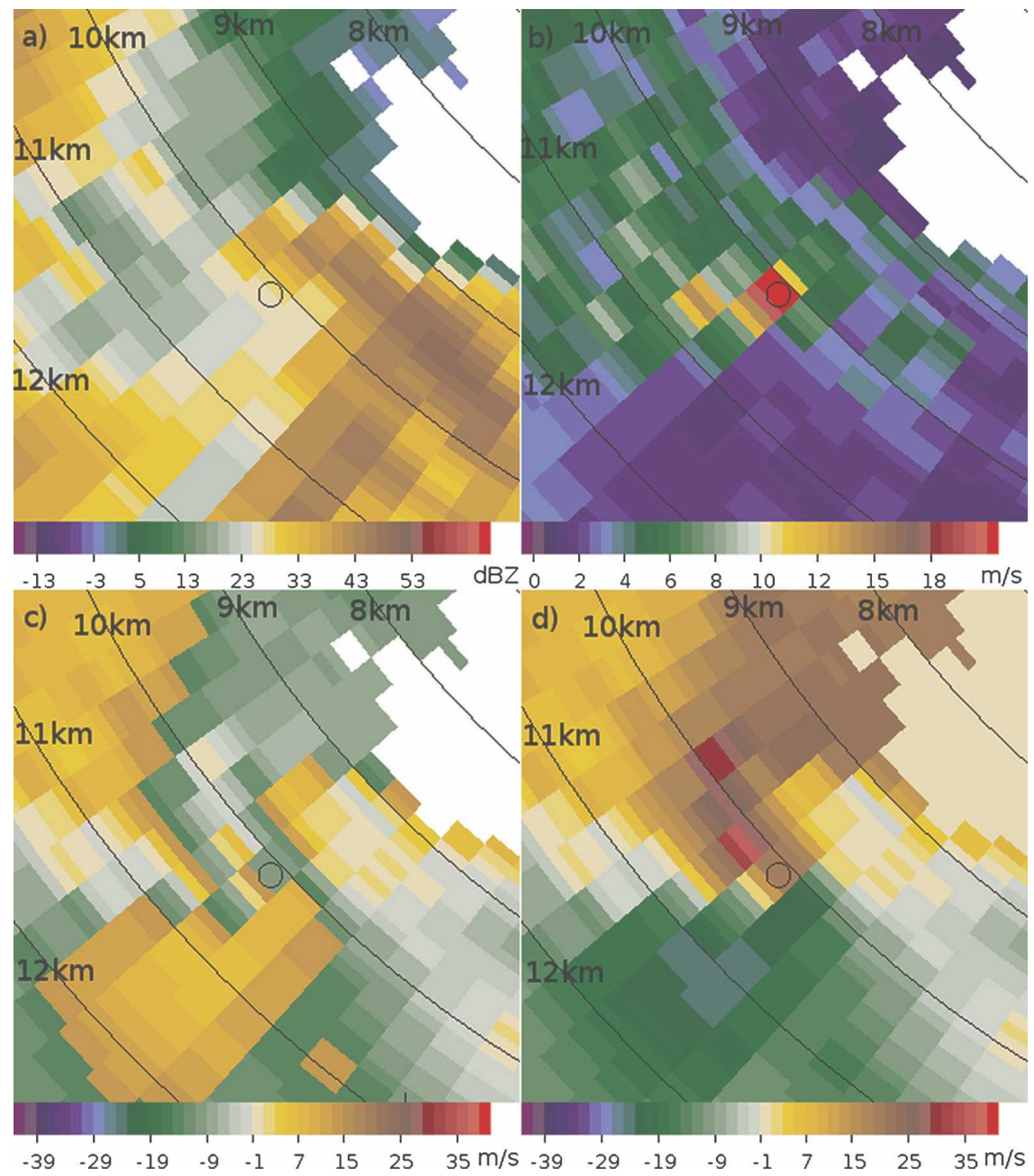

FIG. 16. As in Fig. 14, but for a radar located $10 \mathrm{~km}$ from the tornado.

power in EXP8 (Fig. 12a) to that in CNTL (Fig. 3a) reveals the effects of Rayleigh attenuation at $\mathrm{X}$ band. On the radar side of the storm, the X-band returned power is approximately $10 \mathrm{~dB}$ greater than that of the $\mathrm{S}$ band (Fig. 13), as expected from the dependence of returned power on the transmitted wavelength [Eq. (16)]. On the opposite side of the storm from the radar, this difference decreases to $\sim 3 \mathrm{~dB}$, corresponding to $\sim 7 \mathrm{~dB}$ decrease in the returned power at $\mathrm{X}$ band due to Rayleigh attenuation. As previously mentioned, the Rayleigh approximation underestimates attenuation at shorter wavelengths, so the actual attenuation at $\mathrm{X}$ band for such a storm would be much greater. Here we are merely demonstrating the ability of the emulator to properly handle the propagation effects. The current algorithm could easily incorporate extinction cross sections from Mie (1908) or T-matrix (Waterman 1965) calculations. In addition to non-Rayleigh attenuation, the model does not include ice microphysics. Hence, wet hail, a strong attenuator (Battan 1971), is not included.

Another significant difference between $\mathrm{S}$ band and $\mathrm{X}$ band is the amount of velocity aliasing (Fig. 12c). At X band, the Nyquist velocity for a given PRF is 30\% of that at S band; in EXP8, the Nyquist velocity is 11.25 $\mathrm{m} \mathrm{s}^{-1}$. Consequently, the EXP8 Doppler velocity field shows a large amount of aliasing, with some regions, such as the storm's mesocyclone, exhibiting aliasing by more than one Nyquist interval.

\section{Application to tornado detection}

To illustrate the research and operational value of the radar emulator, the detectability of tornadic signatures is examined as a function of the radar range from the tornado and the azimuthal sampling interval. The emulated radar characteristics (Table 3 ) follow those of the Integrated Project 1 (IP1) radars deployed by the 


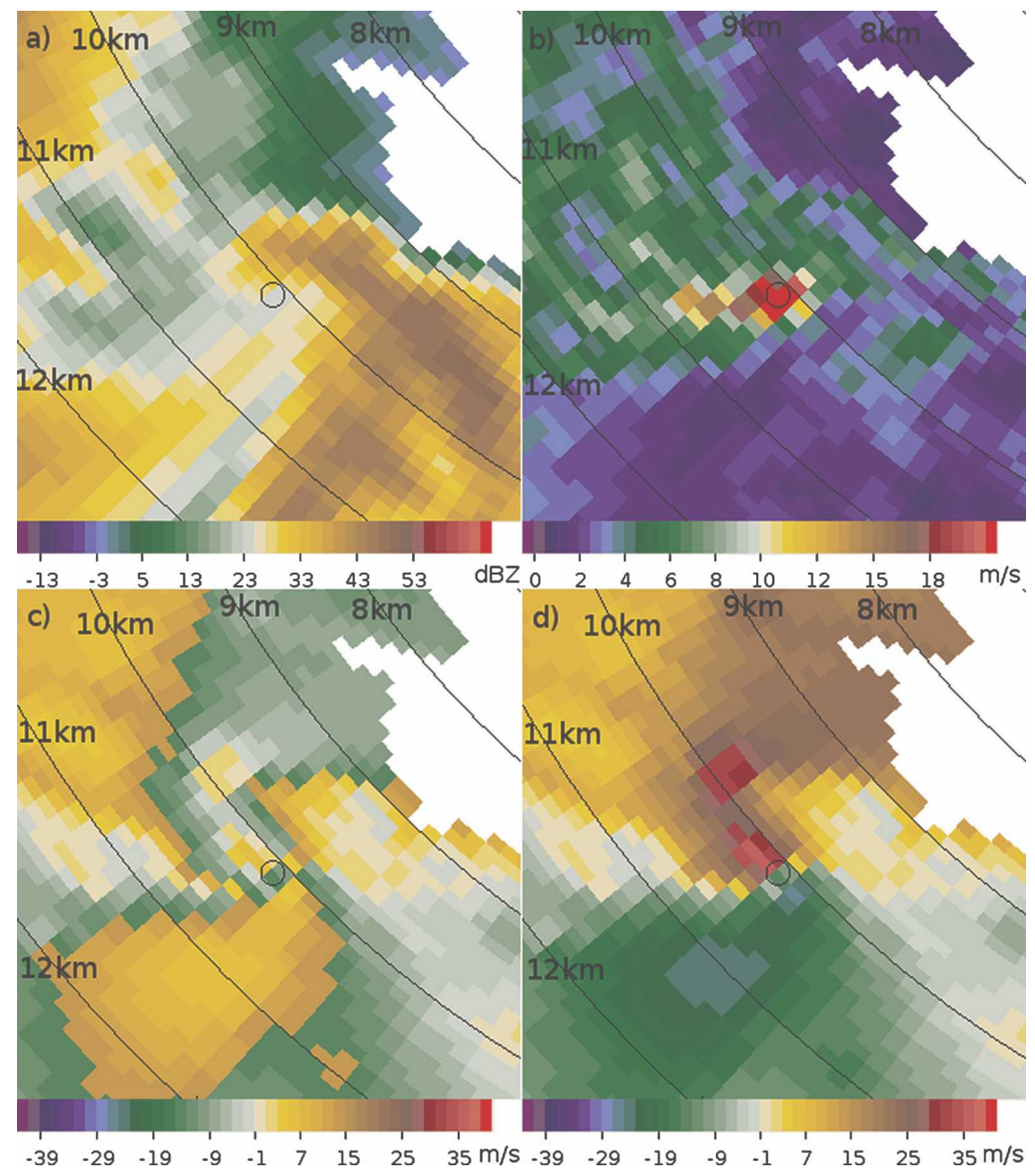

FIG. 17. As in Fig. 16, but for a radar azimuthally oversampling by a factor of 2 .

CASA Engineering Research Center (Brotzge et al. 2005). To keep cost low, these radars have a broad beam ( $2^{\circ}$ half-power beamwidth), use relatively low power $(25 \mathrm{~kW})$, and operate at $\mathrm{X}$ band. One of the goals of CASA is to improve the detection of low-level hazardous weather, such as tornadoes, by placing the radars close to each other and by performing collaborative adaptive sampling of the lowest $3 \mathrm{~km}$ of the atmosphere. The average radar spacing of the IP1 network is about $30 \mathrm{~km}$.

Using the known location and intensity of the tornado in the model as a baseline, this study examines the values of several tornado intensity metrics, including maximum velocity $V_{\max }$, maximum radial velocity difference $\Delta V$, diameter $D$, and axisymmetric vorticity $\zeta_{a}$, as determined directly from the emulated radial velocity data. They are examined as functions of range $(3,10$, 30 , and $50 \mathrm{~km}$ ) from the tornado, using both azimuthally matched sampling ( $2^{\circ}$ intervals) and oversampling (at $1^{\circ}$ intervals). The axisymmetric vorticity, defined as the vorticity for an axisymmetric vortex having the same $\Delta V$ and diameter as the tornado, is given by

$$
\zeta_{a}=\frac{2 \Delta V}{D}
$$

The intensity parameters are used to quantify the range dependency of tornado detection by $2^{\circ}$ beam X-band radars. To eliminate the impact of dealiasing algorithms, this quantitative analysis assumes perfectly dealiased Doppler velocities and, hence, represents the best-case scenario. Furthermore, the known location of the tornado is used to choose the gates for the calculation of the parameters, as opposed to choosing a location based on the position of the velocity maxima in the data. The values of these parameters for all cases, with different range and azimuthal sampling combinations, are listed in Table 4. It should also be noted that the 

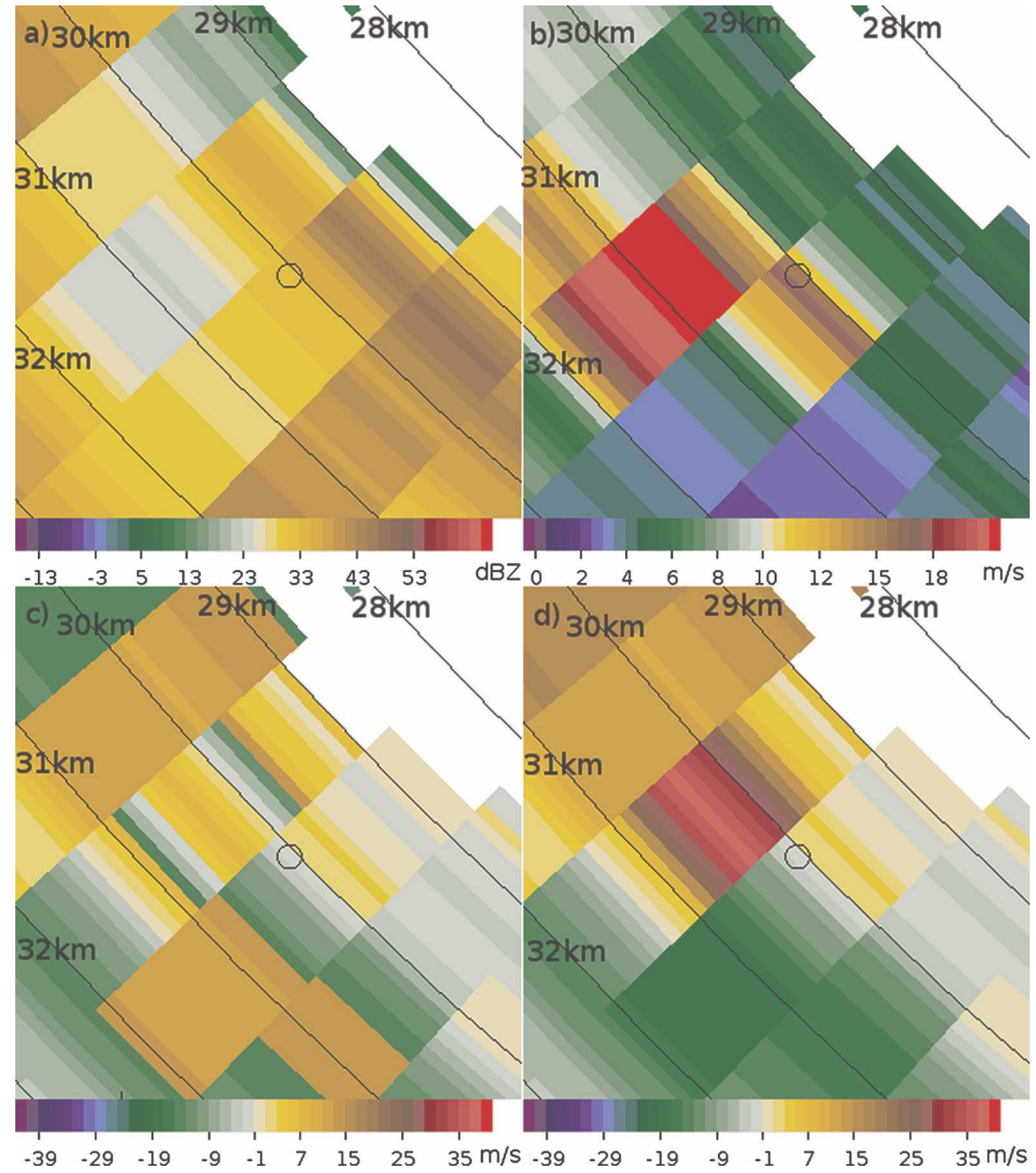

FIG. 18. As in Fig. 14, but for a radar located $30 \mathrm{~km}$ from the tornado.

viewing angle chosen here (from northeast) minimizes the impact of attenuation at $\mathrm{X}$ band.

At 3-km range, the tornado can be clearly identified in both the matched and oversampled moment data (Figs. 14 and 15). The tornado resides in the tip of a well-defined hook echo in a region of enhanced spectrum width. At this range, the strong flow within the tornado can be diagnosed even in the aliased Doppler radial velocity field, especially when the storm is azimuthally oversampled by the radar beam. Indeed, the oversampled unaliased velocity field (Fig. 15d) shows separated inbound and outbound velocity maxima in the tornado vortex. Separation between maximum radial velocities is a useful criterion for resolving a tornadic circulation. Even at this close range, however, the peak Doppler velocity of the tornado measured by the radar (Table 4) is greatly decreased from the true value of $78 \mathrm{~m} \mathrm{~s}^{-1}$. The distance between peak Doppler velocities, $216 \mathrm{~m}$, agrees well with the $\sim 200 \mathrm{~m}$ distance between velocity maxima in the model field. Axisymmetric vorticities of 0.864 and $1.024 \mathrm{~s}^{-1}$ for the matched and oversampled cases, respectively, further indicate that the tornadic circulation is well resolved by the $2^{\circ}$ half-power beamwidth radar at 3-km range.

Moving the radar to $10 \mathrm{~km}$ away from the tornado resulted in degradation of the radar-derived structure as the geometric width of the beam increased (Figs. 16 and 17). While the hook echo and maxima in spectrum width were still fairly well resolved in the matched sampling case, the peak Doppler velocities associated with the tornado were located much farther apart $(705 \mathrm{~m})$, with the peak velocity down to $35.2 \mathrm{~m} \mathrm{~s}^{-1}$. Consequently, the estimated vorticity decreased to $0.163 \mathrm{~s}^{-1}$, or $20 \%$ of the value obtained at $3-\mathrm{km}$ range. It should be noted that the peak inbound velocity measured with the tornado was only $22.4 \mathrm{~m} \mathrm{~s}^{-1}$, less than the 24.1 $\mathrm{m} \mathrm{s}^{-1}$ inbound velocity associated with the mesocyclone. It is only when the storm was azimuthally over- 


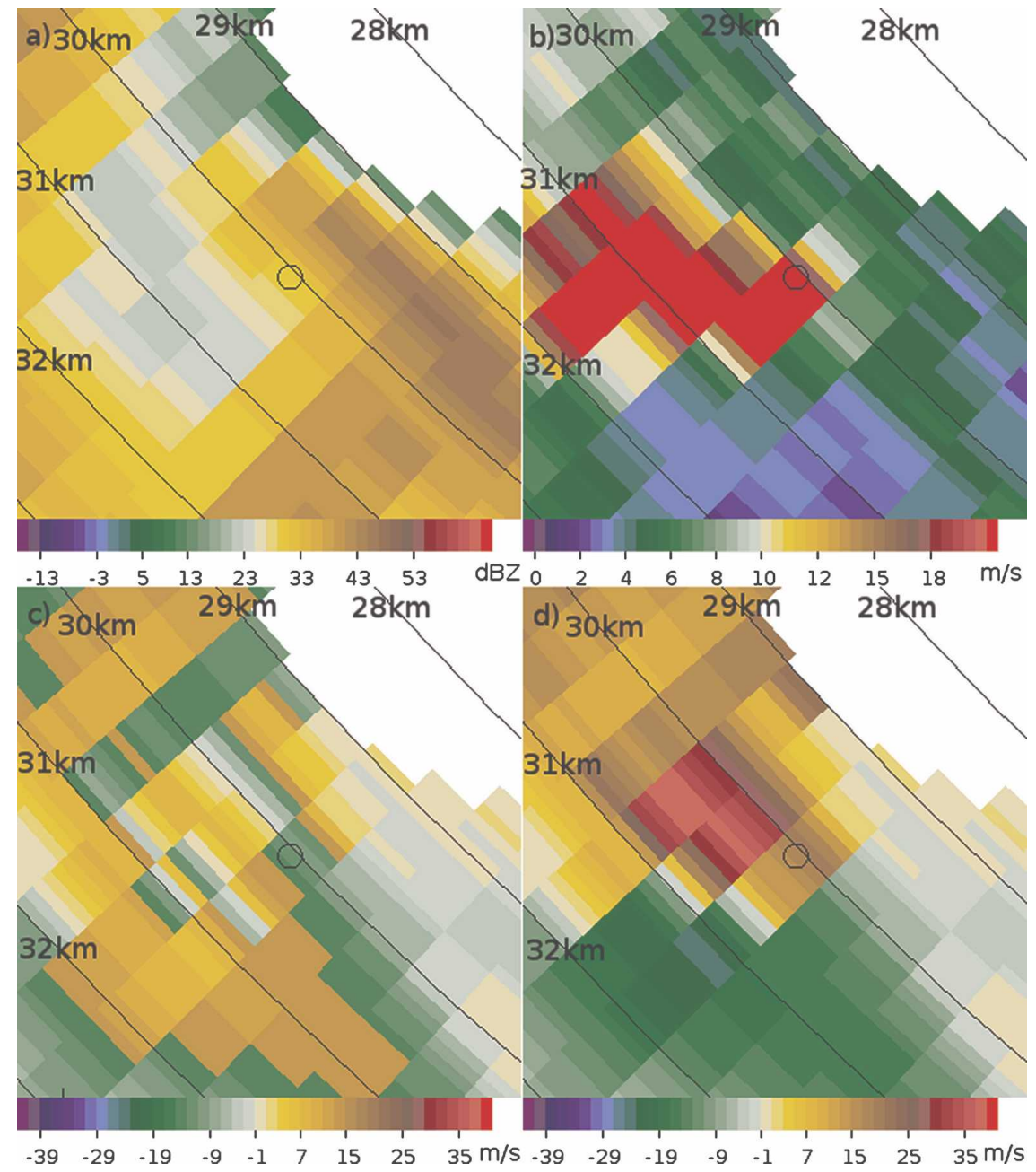

FIG. 19. As in Fig. 18, but for a radar azimuthally oversampling by a factor of 2 .

sampled that the tornadic circulation was resolved at $10-\mathrm{km}$ range with the current radar system. With $1^{\circ}$ azimuthal sampling (Fig. 17), the distance between the velocity maxima decreased to $529 \mathrm{~m}$, which was the main factor for the increase in the axisymmetric vorticity to $0.237 \mathrm{~s}^{-1}$.

At $30-\mathrm{km}$ range with matched sampling, the structure of the hook echo and spectrum width field is so degraded that the location of the tornado is no longer well diagnosed by the radar parameters (Fig. 18). Even the nonaliased velocity field no longer shows separate velocity maxima for the tornado and mesocyclone. Instead, a single maximum is located several gates away from the known location of the tornado. The tornadoscale flow is no longer resolved because the half-power beamwidth is over $1 \mathrm{~km}$ wide at this range, roughly 5 times the diameter of the tornado. Also at this range, the poor resolution of the data makes distinguishing storm shear from regions of aliased velocity a challenge
(Fig. 18c). Using the known location of the tornado, a vorticity estimate of $0.064 \mathrm{~s}^{-1}$ is calculated for this circulation. Such a low vorticity estimate would likely not be associated with a strong tornadic mesocyclone. It should be noted that the inbound velocity measured and used in the calculation is only $1.6 \mathrm{~m} \mathrm{~s}^{-1}$. Even when oversampling is performed (Fig. 19) the tornadic circulation is still not resolved by a $2^{\circ}$ beamwidth radar when it is located $30 \mathrm{~km}$ away. While the separation between the maximum inbound and outbound velocities in the mesocyclone and a region of enhanced spectrum width exists, there is no indication of a tornado vortex signature (Brown et al. 1978). Furthermore, dealiasing the $\mathrm{X}$-band radial velocity field becomes challenging, as the beam containing the tornado appears to be embedded in a broad-scale region of aliased inbound velocities. In reality, the minimum in radial velocity associated with the tornado separates aliased inbound velocities from the true receding flow. 

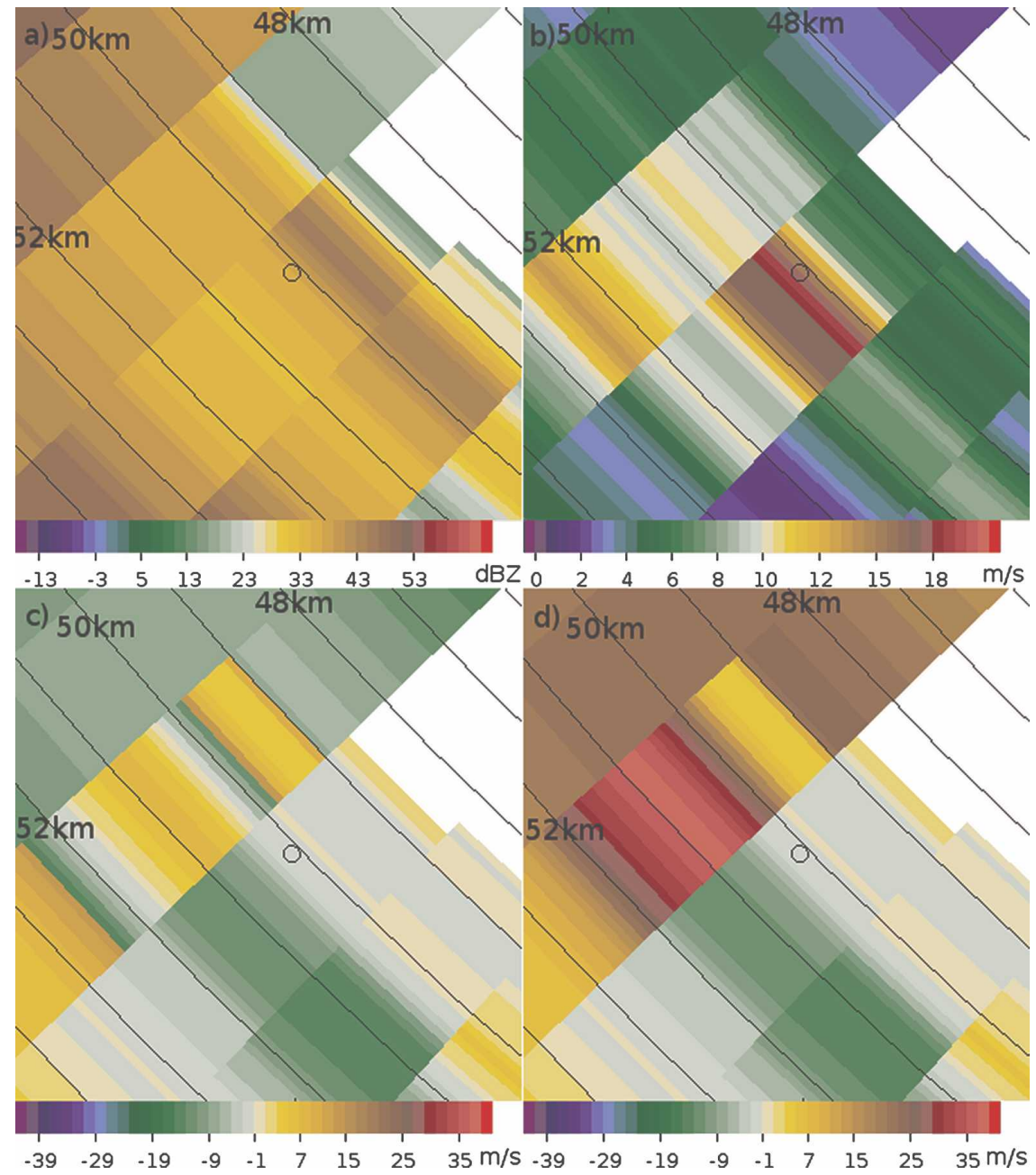

FIG. 20. As in Fig. 14, but for a radar located $50 \mathrm{~km}$ from the tornado.

At 50-km range, the half-power beamwidth is $1.7 \mathrm{~km}$ across, reducing even the mesocyclone-scale circulation to gate-to-gate shear and completely obscuring the tornado-scale flow (Figs. 20, 21). It would be very difficult to detect reliably a tornado with the size and intensity as in the simulation using only the moment data at this range.

\section{Conclusions}

A Doppler radar emulator based on Rayleigh scattering has been developed that simulates a wide range of radar operating characteristics, including range and azimuthal oversampling, velocity and range aliasing, Rayleigh attenuation, second-trip echoes, antenna sidelobes, and anomalous propagation. The emulator calculates returned power, equivalent radar reflectivity factor, Doppler velocity, and Doppler spectrum width from cloud model output containing fields of wind, temperature, moisture, and hydrometeor species. The capabilities of the emulator are demonstrated using a high-spatial-resolution simulation of a tornado embedded within a supercell thunderstorm. It is shown that the emulator is a useful tool for evaluating the capabilities and trade-offs in the design, deployment, and operation of radar systems. Given that the emulator can produce numerous synthetic datasets for a wide range of storm types and radar characteristics, we believe that such a tool can be a significant aid in the development of radar algorithms. Such realistically emulated data can also be used in observing system simulation experiments (OSSEs) such as those of Xue et al. (2006) for examining the potential impact of radar data on thunderstorm analysis and prediction.

Using the output from a 50-m horizontal-resolution simulation of a supercell storm that explicitly resolves 

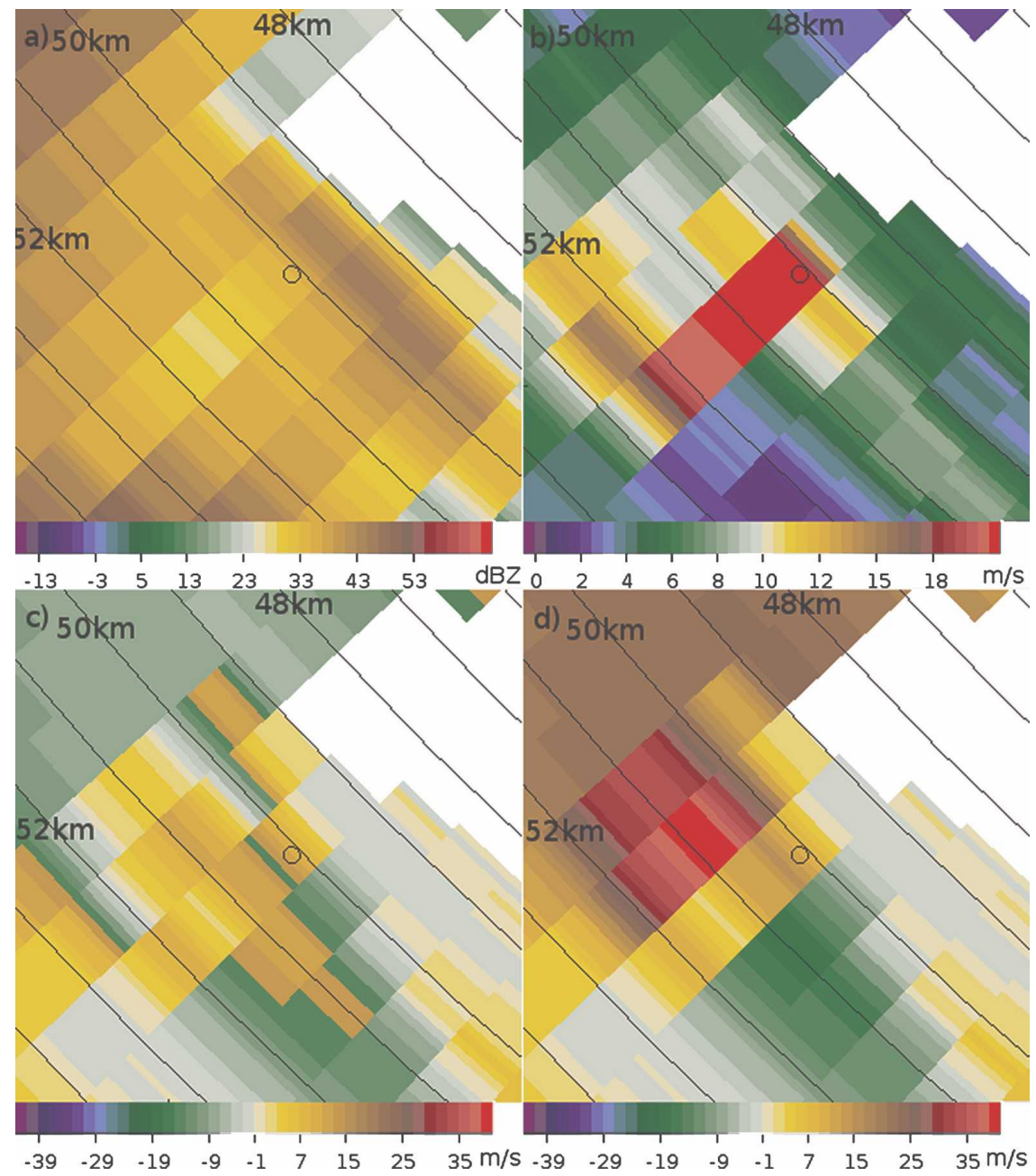

FIG. 21. As in Fig. 20, but for a radar azimuthally oversampling by a factor of 2 .

an F3 intensity tornado of about $200 \mathrm{~m}$ in diameter, the basic capabilities of the emulator are first tested in a set of experiments that examines the effects of radar wavelength, beamwidth, azimuthal oversampling, gate length, sidelobes, pulse repetition frequency, and the effects of velocity and range aliasing. Results consistent with theory are observed from the simulated data.

As an example of the emulator's many potential applications, the detection of the simulated tornado described above by a $2^{\circ}$ half-power beamwidth $\mathrm{X}$-band radar is examined. The emulated data show that the strength of the diagnosed tornado circulation decreases rapidly with range, with the tornado-scale flow becoming unresolved at and beyond $30 \mathrm{~km}$. Azimuthal oversampling improves the ability to diagnose the tornado vortex, especially from the $10-$ to $30-\mathrm{km}$ ranges. At shorter ranges, the $2^{\circ}$ beam-matched azimuthal sampling is sufficient. It is important to note that the simulated tornado examined here represents the top $10 \%$ of tornadoes occurring in nature in terms of intensity. The much more prevalent weaker and/or smaller tornadoes will be even harder to detect.

A significant problem demonstrated by the emulator is the impact of velocity aliasing at $\mathrm{X}$ band on the potential diagnosis of the circulations. Correct dealiasing is crucial to tornado detection when the detection algorithm mainly relies on the radial velocity data (e.g., Burgess et al. 1993; Liu et al. 2007). Any method that can increase the effective Nyquist velocity, such as the use of staggered PRT (Gray et al. 1989), would likely be helpful.

Future studies will include more radar operating parameters as well as the use of objective algorithms to evaluate tornado detection for broad-beam X-band radars. This work is motivated by the Integrated Project I (IP1) of the Collaborative Adaptive Sensing of the Atmosphere (CASA; Brotzge et al. 2005) Engineering Research Center that recently deployed four $2^{\circ}$ half- 
power beamwidth X-band radars in central Oklahoma. One of the goals of such inexpensive networks is tornado detection, based on the promise of being able to observe at low altitudes $(0-3 \mathrm{~km})$ and at short ranges (less than $30 \mathrm{~km}$ ), and do so in a collaborative and adaptive manner. In the future, the radar emulator will be further enhanced to include Mie scattering, as well as scattering from ice-phase hydrometeors, and the antenna routine will be modified to allow for emulation (using many model time steps) of electronically steered phased-array radars that can point the beam in arbitrary directions on a pulse-to-pulse basis. This will enable applied research in the phased array radar (Forsyth et al. 2005) program and further enhance the educational utility of the radar emulator.

Acknowledgments. This work was supported by graduate research fellowships sponsored by the Office of Naval Research through the American Meteorological Society and by the Army Research Office through the National Defense Science and Engineering Graduate Fellowship program. Partial support was also provided by NSF Grant EEC-0313747 to the CASA ERC. M. Biggerstaff was supported by NSF Grants ATM0619715, ATM-0410564, and ATM-0618727, while M. Xue was supported by NSF Grants ATM-0129892, ATM-0331594, ATM-0331756, and ATM-0530814. The authors would also like to thank two anonymous reviewers whose many comments helped improve the quality of this work.

\section{REFERENCES}

Battan, L. J., 1971: Radar attenuation by wet ice spheres. J. Appl. Meteor., 10, 247-252.

1973: Radar Observation of the Atmosphere. University of Chicago Press, 324 pp.

Bean, B. R., and E. J. Dutton, 1966: Radio Meteorology. National Bureau of Standards Monogr., No. 92, U.S. Government Printing Office, $435 \mathrm{pp}$.

Brandes, E. A., G. Zhang, and J. Vivekanandan, 2002: Experiments in rainfall estimation with a polarimetric radar in a subtropical environment. J. Appl. Meteor., 41, 674-685.

Brotzge, J. A., K. Brewster, B. Johnson, B. Philips, M. Preston, D. Westbrook, and M. Zink, 2005: CASA's first testbed: Integrated project \#1. Preprints, 32nd Conf. on Radar Meteorology, Albuquerque, NM, Amer. Meteor. Soc., 14R.2.

Brown, R. A., L. R. Lemon, and D. W. Burgess, 1978: Tornado detection by pulsed Doppler radar. Mon. Wea. Rev., 106, 29-38.

— , V. T. Wood, and D. Sirmans, 2002: Improved tornado detection using simulated and actual WSR-88D data with enhanced resolution. J. Atmos. Oceanic Technol., 19, 17591771.

Burgess, D. W., R. J. Donaldson Jr., and P. R. Desrochers, 1993: Tornado detection and warning by radar. The Tornado: Its
Structure, Dynamics, Prediction, and Hazards, Geophys. Monogr., Vol. 79, Amer. Geophys. Union, 203-221.

Capsoni, C., and M. D'Amico, 1998: A physically based radar simulator. J. Atmos. Oceanic Technol., 15, 593-598.

- - _ , and R. Nebuloni, 2001: A multiparameter polarimetric radar simulator. J. Atmos. Oceanic Technol., 18, 1799-1809.

Chandrasekar, V., and V. N. Bringi, 1987: Simulation of radar reflectivity and surface measurements of rainfall. J. Atmos. Oceanic Technol., 4, 464-478.

Crum, T. D., and R. L. Alberty, 1993: The WSR-88D and the WSR-88D operational support facility. Bull. Amer. Meteor. Soc., 74, 1669-1687.

Doviak, R. J., and D. S. Zrnić, 1993: Doppler Radar and Weather Observations. $2 \mathrm{~d}$ ed. Academic Press, 562 pp.

Forsyth, D. E., and Coauthors, 2005: The National Weather Radar Testbed (phased-array). Preprints, 32nd Conf. on Radar Meteorology, Albuquerque, NM, Amer. Meteor. Soc., 12R.3.

Fujita, T. T., 1971: Proposed characterization of tornadoes and hurricanes by area and intensity. SMRP Research Rep. 91, University of Chicago, $15 \mathrm{pp}$.

Gray, G., B. Lewis, J. Vinson, and F. Pratte, 1989: A real-time implementation of staggered PRT velocity unfolding. J. Atmos. Oceanic Technol., 6, 186-187.

Kerr, D. E., 1951: Propagation of Short Radio Waves. McGrawHill, 728 pp.

Kessler, E., 1969: On the Distribution and Continuity of Water Substance in Atmospheric Circulations. Meteor. Monogr., No. 32, Amer. Meteor. Soc., 84 pp.

Krajewski, W. F., R. Raghavan, and V. Chandrasekar, 1993: Physically based simulation of radar rainfall data using a space-time rainfall model. J. Appl. Meteor., 32, 268-283.

Lemon, L. R., and C. A. Doswell III, 1979: Severe thunderstorm evolution and mesocyclone structure as related to tornadogenesis. Mon. Wea. Rev., 107, 1184-1197.

Lewellen, W. S., D. C. Lewellen, and R. I. Sykes, 1997: Largeeddy simulation of a tornado's interaction with the surface. $J$. Atmos. Sci., 54, 581-605.

Liu, S., M. Xue, and Q. Xu, 2007: Using wavelet analysis to detect tornadoes from Doppler radar radial-velocity observations. $J$. Atmos. Oceanic Technol., 24, 344-359.

Marshall, J. S., and W. M. Palmer, 1948: The distribution of raindrops with size. J. Atmos. Sci., 5, 165-166.

Mie, G., 1908: Beigrade zur Optik truber Medien, speziell kolloidaler Metallosungen. Ann. Phys., 25, 377-445.

Ray, P. S., B. Johnson, K. W. Johnson, J. S. Bradberry, J. J. Stephens, K. K. Wagner, R. B. Wilhelmson, and J. B. Klemp, 1981: The morphology of several tornadic storms on 20 May 1977. J. Atmos. Sci., 38, 1643-1663.

Ryde, J. W., and D. Ryde, 1945: Attenuation of centimeter and millimeter waves by rain, hail, fogs, and clouds. General Electric Co. Rep. 8670.

Saxton, J. A., 1946: Meteorological Factors in Radio-Wave Propagation. Physical Society, 325 pp.

Ulbrich, C. W., 1983: Natural variations in the analytical form of the raindrop size distribution. J. Appl. Meteor., 22, 17641775.

Waterman, P. C., 1965: Matrix formulation of electromagnetic scattering. Proc. IEEE, 53, 805-812.

Wicker, L. J., and R. B. Wilhelmson, 1995: Simulation and analysis of tornado development and decay within a threedimensional supercell thunderstorm. J. Atmos. Sci., 52, 2675 2703.

Wood, V. T., and R. A. Brown, 1997: Effects of radar sampling on 
single-Doppler velocity signatures of mesocyclones and tornadoes. Wea. Forecasting, 12, 928-938.

— $-\longrightarrow$, and D. Sirmans, 2001: Technique for improving detection of WSR-88D mesocyclone signatures by increasing angular sampling. Wea. Forecasting, 16, 177-184.

Xue, M., K. K. Droegemeier, V. Wong, A. Shapiro, and K. Brewster, 1995: ARPS version 4.0 user's guide. 380 pp. [Available online at http://www.caps.ou.edu/ARPS.]

- - - , and - , 2000: The Advanced Regional Prediction System (ARPS)-A multiscale nonhydrostatic atmospheric simulation and prediction tool. Part I: Model dynamics and verification. Meteor. Atmos. Phys., 75, 161-193.

, and Coauthors, 2001: The Advanced Regional Prediction System (ARPS) - A multiscale nonhydrostatic atmospheric simulation and prediction tool. Part II: Model physics and applications. Meteor. Atmos. Phys., 76, 143-166.

_- M. Tong, and K. K. Droegemeier, 2006: An OSSE framework based on the ensemble square root Kalman filter for evaluating the impact of data from radar networks on thunderstorm analysis and forecasting. J. Atmos. Oceanic Technol., 23, 46-66.

—, S. Liu, and T-Y. Yu, 2007: Variational analysis of oversampled dual-Doppler radial velocity data and application to the analysis of tornado circulations. J. Atmos. Oceanic Technol., 24, 403-414.

Zrnić, D. S., 1975: Simulation of weatherlike Doppler spectra and signals. J. Appl. Meteor., 14, 619-620. 